\title{
Posters
}

\section{Posters for Health Information Systems}

\author{
Coronary tree modeling with iterative function systems using a genetic algorithm \\ JA Lopez, CD Nugent, ND Black \\ Medical Informatics, Faculty of Informatics, University of Ulster at Jordanstown, Northern Ireland
}

Abstract: In this work the applicability of genetic algorithms (GA) is described for 2D type images from coronary trees modeled by means of an iterative function system (IFS) structure. Strong correlations between elements of the IFS transformation matrix and morphologic characteristics in the angiographic images (2D coronary tree images) are also demonstrated. The characterization of angiographic images trough IFS structures can be seen with foresight to be very helpful in the classification and diagnosis of pathologies associated with myocardial and/or coronary tree morphological disorders.

Introduction and Methods: GA are search and optimization procedures inspired by natural selection and genetics in which the fittest individuals from the population tend to survive and reproduce through many generations [1]. The solution domain for IFS transformation matrixes is vast; hence a GA approach is very powerful to search for applicable functions [2]. The IFS equations are powerful tools in modeling self-contained 2D structures. A relevant issue regarding this kind of structure is its recursive properties. Such properties can simulate non-linear events with few components in a transformation matrix. The number of equations and matrix component of an IFS depends, firstly, on the object dimension (2D, 3D, etc.), secondly, on the image complexity (quantity of spatial transformations) and thirdly and finally on the object fractal dimension. Figure 1 indicates an exemplar angiographic image. Although the contrast liquid gives visual evidence of the coronary tree structure, the image is still not useful to the purposed methodology. Due to this, binarization of the image is necessary using edge filters to obtain the image illustrated in Figure 2. This binary image is the starting point and can be considered as the target for the fitness definition in the GA.

For the IFS structure, a bi-dimensional equation system $(\mathrm{X}, \mathrm{Y})$ and four spatial transformations $(1,2,3$ and 4) were chosen. The IFS structure can be defined as,

$$
\begin{aligned}
& X_{1}=A_{i} \cdot X+B_{i} \cdot Y+C_{i} \\
& Y_{1}=D_{i} \cdot X+E_{i} \cdot Y+F_{i}
\end{aligned}
$$




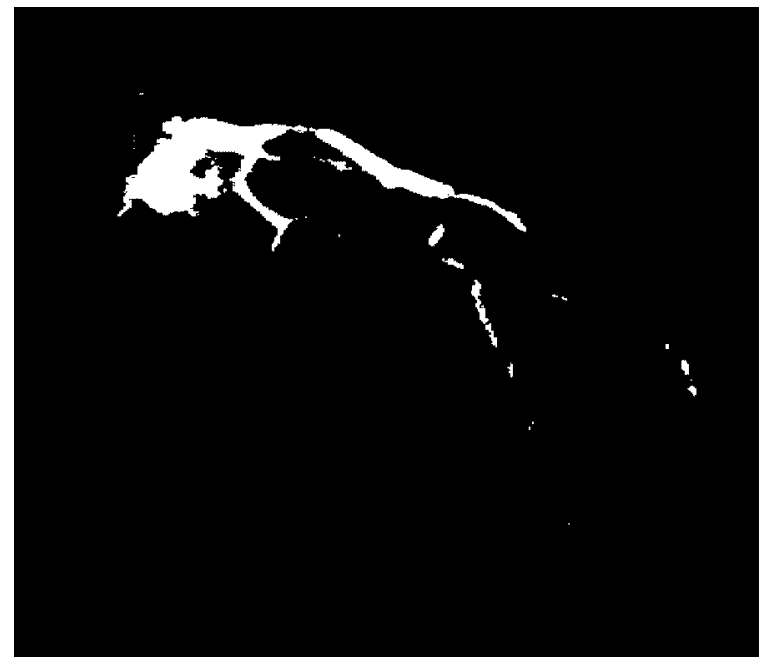

Figure 1. Angiographic image.

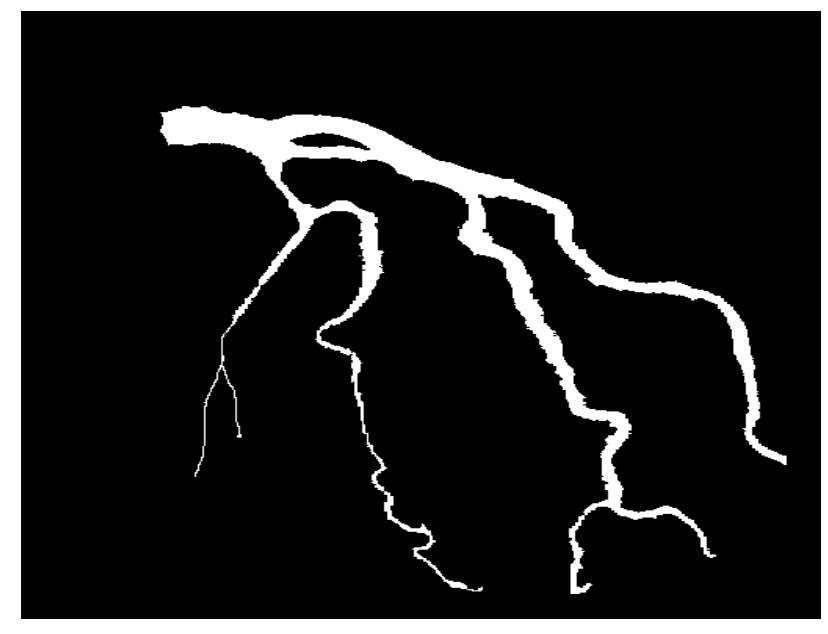

Figure 2. Binarized image (target).

where $i=\{1,2,3$ and 4$\}$ and represents the associated spatial transformation. With this, the transformation matrix can be defined as follows:

$$
\left(\begin{array}{llllll}
A_{1} & B_{1} & C_{1} & D_{1} & E_{1} & F_{1} \\
A_{2} & B_{2} & C_{2} & D_{2} & E_{2} & F_{2} \\
A_{3} & B_{3} & C_{3} & D_{3} & E_{3} & F_{3} \\
A_{4} & B_{4} & C_{4} & D_{4} & E_{4} & F_{4}
\end{array}\right)
$$

The above transformation matrix characterizes the fractal image in the Cartesian plane and will be the "individual" in the GA. The problem at this stage is to find the best model for the transformation matrix of the coronary tree. The fitness function employed during the GA search was as follows,

$$
\text { Fitness }=1-\frac{\left|m a x \_p i x e l-m a t c h \_p i x e l\right|}{m a x \_p i x e l}
$$

where max_pixel represents the number of pixels required to match the actual coronary tree (target) and match_pixel is the number of pixels generated by the individual or current transformation matrix (3), which matches the target (Figure 2). With this, the fitness function will take a value of 1 for the desired individual and the higher the value of max_pixel, the better the representation of the IFS synthesized coronary tree. It is desirable, however, to find a tradeoff between max_pixel and the search time of the GA.

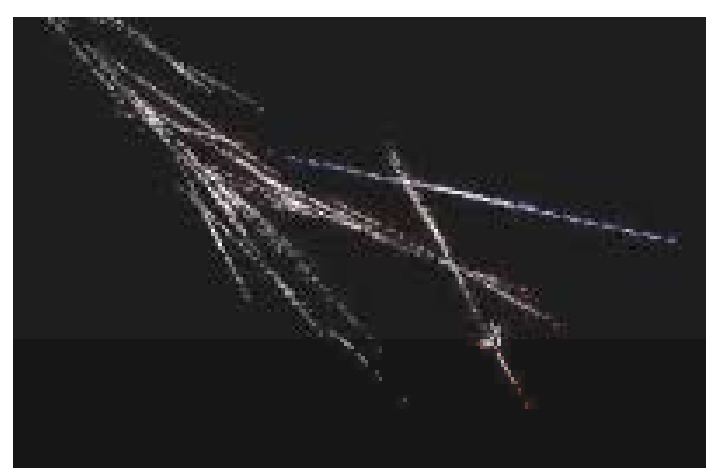

Fig. 3: Synthesized coronary. 
Results and Conclusions: Figure 3 presents a synthesized image that corresponds to the transformation matrix produced. The GA search had a runtime of 12 hours using a $233 \mathrm{MHz}$ i386 type processor. The max_pixel constant for this run was set to be 100.000 matching pixels in the synthesized tree. With this, it has been demonstrated that for relatively low values of max_pixel, a clear likeness with the real coronary tree begins to appear. With such an approach, observations of strong correlations between specific morphologic characteristics of the image and the variation of such elements were recorded. In conclusion, the modeling method by IFS structures promise to be very powerful in coronary trees and this modeling technique results in a helpful tool for the diagnosis and/or classification of pathologies associated with the external morphology of the heart and the coronary arteries.

References

[1] D. E. Goldberg, Genetic Algorithm in Search, Optimization, and Machine Learning. Reading, MA: Addison-Wesley (1989).

[2] Boyeon Kim, Kwangsuk Park. A study on the modeling of nonlinear system using genetic programming. Proc. $18^{\text {th }}$ Int. Conference - IEEE/EMBS. Amsterdam. (1996).

\title{
An electro-oculogram based human-computer interface for the disabled
}

\author{
J.A. Lopez ${ }^{\mathrm{a}}$, C.D. Nugent ${ }^{\mathrm{a}}$, K.I. Ziri-Castro ${ }^{\mathrm{b}}$, N.D. Black ${ }^{\mathrm{a}}$ \\ ${ }^{a}$ Medical Informatics, Faculty of Informatics, University of Ulster at Jordanstown, Northern Ireland \\ ${ }^{\mathrm{b}}{ }^{\dagger}$ Northern Ireland BioEngineering Centre, University of Ulster at Jordanstown, Northern Ireland
}

Abstract: A human-computer interface is described that allows patients with severe neuro-motor disabilities to move a pointer on the screen of a personal computer (PC) using an electro-oculogram (EO) interface. The pointer is driven through the processing of 3 discrete ocular movements (right, left and up) obtained non-invasively from the patient. The system is cost effective and highly efficient in the control of graphical applications by persons suffering limitations of the upper and lower limbs. Evaluation on a cohort of 15 users showed high levels of acceptance and usability.

Introduction and Methods: Severe neuro-motor deficiencies such as quadriplegia do not permit mentally active individuals to communicate and develop tasks associated with motor abilities. Individuals in this situation could be re-incorporated into society if there were a way to take advantage of their limited motor capacity and become then productive by means of a support system such as a PC. To address these needs, an interface that permits the management of a mouse pointer on a PC screen has been developed. The movement of the pointer is controlled by processing a person's EO signal. The EO itself is generated from stationary potentials generated between the cornea and the retina in the eye. This stationary dipole can be used to measure or to sense the position of the eye following acquisition with electrodes located at both sides, above and below the eye socket. When sight attention remains fixed, the stationary eye dipole locates itself symmetrically between two opposite electrodes

hence the EO signal is zero. If for example the sight is directed towards the left, the cornea, which possess a positive charge, ensures the left most electrode is more positive. In the same way, this phenomenon exists in electrodes located above and below the eye which register vertical axis movements [1]. A block diagram of the interface developed is shown in Figure 1. The instrumentation amplifiers have a gain of 3000 and cut of frequencies of 3 and $10 \mathrm{~Hz}$. This bandwidth permits high integrity detection of long swing eye movements with eventual eye blinks, without DC component interference. 


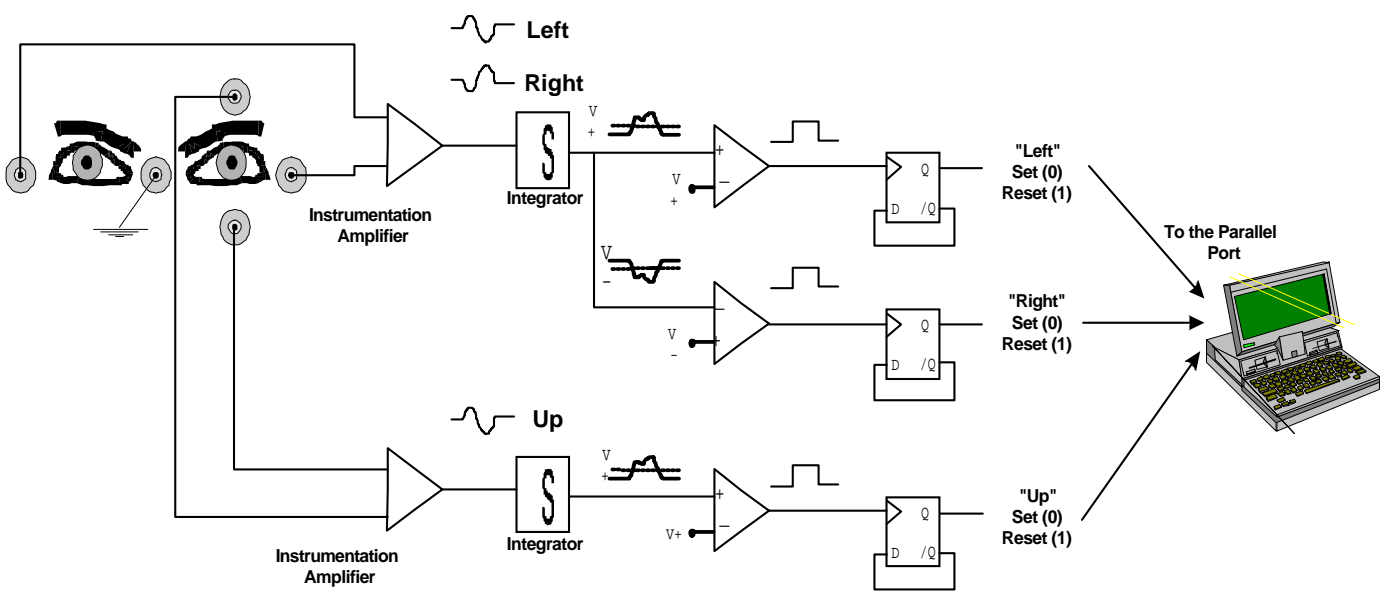

Fig. 1: Block diagram of the EO human-computer interface.

\begin{tabular}{|c|c|c|c|}
\hline Task & $\begin{array}{c}\text { PE } \\
{[\mathrm{mov}]}\end{array}$ & $\begin{array}{l}\text { ST } \\
\text { [s] }\end{array}$ & $\begin{array}{c}\text { SA } \\
{\left[\mathrm{mov} \mathrm{s}^{-1}\right]}\end{array}$ \\
\hline WWW Search & 13.2 & 48.3 & 0.273 \\
\hline Phone Number Dialling & 12.8 & 35.2 & 0.367 \\
\hline
\end{tabular}

Table 1: Measurements for the two monitored tasks.

For a left direction eye movement, the signal has a phase of zero degrees and for right movement, the phase is $180^{\circ}$. To detect the direction of eye movement, following amplification and filtering, the signal is integrated and compared to accomplish phase detection. Two comparators are employed to either detect right and left movement through the production of excitatory signals. Direction of eye movement in the vertical plane is detected in a similar manner. The final stage is formed by D-type flipflops. These permit, with one discrete eye movement, the setting and resetting of a signal. With 3 flipflops, 8 different combinations are possible permitting 8 different pointer movements. The outputs from the flip-flops are connect to the PC via the parallel port.

Results: The system has been tested with 15 patients. Each found it to be user friendly, easy to use and quick to learn. The mean effective learning time, while patients were fed with a feedback signal protocol was 22 minutes. Two specific tasks were used; an Internet search and the dialling of a telephone number using a virtual keyboard. Table 1 shows results for Process Efficiency (PE), Selection Time (ST) and Selection Average (SA) for each task.

Conclusions: A non-invasive device that allows individuals with severe disabilities to use the movement of the eye to control a PC pointer in real time has been developed. This device allows individuals, more often young and mentally active, to communicate with the outside world [2] and enjoy the almost infinite possibilities offered by the PC environment: web browsing, document writing, control 
of external devices e-mail etc. Testing on a cohort of 15 patients has resulted with feedback of ease of use and acceptability.

\title{
References
}

[1] Kris, C., "Electro-oculography", in O. Glasser(ed.), Medical Physics, Vol. III, 692-700 (1960).

[2] Mendez, N., Mora, F. And Chang, O. A microprocessor based communication and control system for the severely disabled. IX Congresso Brasileiro de Engenharia Biomedica, Campinas, Brazil, short communication (1985).

\section{A human-computer interface to assist in the development of musical devices for physically impaired students}

\author{
C.D. Nugent ${ }^{\mathrm{a}}$, M. McCormack ${ }^{\mathrm{b}}$, RJ Davies ${ }^{\mathrm{a}}, \mathrm{ND} \mathrm{Black}^{\mathrm{a}}$ \\ ${ }^{\mathrm{a}}$ Medical Informatics, Faculty of Informatics, University of Ulster at Jordanstown, Northern Ireland \\ ${ }^{\mathrm{b} \dagger}$ The Drake Music Ireland Project
}

Abstract: The development of a low cost microcontroller based system to map switch inputs to MIDI format has allowed the development of two innovative musical devices for use by physically impaired musical students. Both devices have been tested on a cohort of users with varying degrees of abilities and high levels of user acceptance and usability have been recorded.

Introduction: The field of electronic music has only existed for under a century, giving the instruments produced little time to mature. Technology is developing in such a rapid fashion that new synthesis methods and capabilities rapidly displace those of only a few years before [[1]]. The design of appropriate interfaces is therefore in a continual state of evolution: always driven by new methods of sound generation that enable, and occasionally require, expression and control over new degrees of freedom. Musical interface research has merged with the broader field of human-computer interface (HCI). Such methods offer revolutionary opportunities to advance the quality of life, particularly as the power, functionality and affordability of computers continues to soar [[2]]. With advances in computerised technologies new types of interfaces are required, as users are no longer desk-bound. This is especially the case for physically impaired people, who may be unable to use a keyboard and/or mouse; hence the need for alternative interfaces is compelling.

It has been the aim of this study to develop a MIDI based platform which can be used as the basis of access technologies for physical impaired people who wish to play music.

Methods: The main objective of the development of the platform was to provide an instrumental control interface to enhance real-time performance possibilities for musicians with physical disabilities. The process in this instance of playing music was reduced to a simple process of switching notes on or off. Hence, a low cost microcontroller based system was developed with the ability to poll up to 16 switch inputs and subsequently map these to MIDI events. Providing a MIDI compatible output both in terms of hardware and software requirements results in a platform which is compatible with any MIDI based sound module. The development of such a platform provides the basis, in electronic terms, for the development of new approaches in access technologies for musical instruments for physically impaired persons.

Results: The aforementioned platform was developed with the central microcontroller PIC16F84. Two innovative musical instruments were developed around this platform by developing suitable electromechanical interfaces. The first device was a 'Giant Keyboard' and provides a user with the ability to play one octave. The keys on the keyboard are approximately 10 times larger than that of a 


\begin{tabular}{|l|l|}
\hline Giant Keyboard & Paddle Player \\
\hline 13 note MIDI controller keyboard & 8 note vertical MIDI controller \\
\hline 16 MIDI channel selector & N/A \\
\hline 3 stage volume control & Full volume control \\
\hline 13-switch controller output & $\begin{array}{l}\text { Pressure sensitive, mappable to } \\
\text { MIDI functions }\end{array}$ \\
\hline
\end{tabular}

Table 1 General specifications of musical devices.

normal keyboard and hence provide usability for persons without full hand movements. The second device was referred to as the 'Paddle Player'. This was a vertically mounted keyboard in which the user pressed keys located on its back surface and the front surface housed a series of illuminative panels, each of which was illuminated proportionally to the pressure exerted on the back switch. Each device was, as previously stated MIDI compatible and connected to a standard sound module. Table 1 outlines the basic specifications of each device.

Conclusions: Based on the development of a platform capable of converting basic switching actions into MIDI format it has been possible with this study to develop two new innovative musical instruments to be used as access technologies for persons with disabilities wishing to undertake real-time musical performance. The devices have been evaluated on a cohort of musical students with varying degree of abilities and all have indicated great acceptance of the devices and expressed comments of ease of use. At present Windows based software being developed for each device to add an extra dimension of a music training tool for users.

\section{References}

[1] Paradiso, J., Electronic Music Interfaces, MIT Media Laboratory Technical Report, pp. 1-22, 1998.

[2] Lipson, E., Warner, D. and Chang, Y, Universal interfacing system for interactive technologies in telemedicine, disabilities, rehabilitation and education, Medicine Meets Virtual Reality, pp. 205-211, 1999.

\section{Quantum informatics}

David Glass and Mark McCartney

Faculty of Informatics, University of Ulster at Jordanstown, Newtownabbey, Co. Antrim, Northern Ireland

E-mail:dh.glass@ulst.ac.uk,m.mccartney@ulst.ac.uk

Abstract: Recent developments in molecular biology have given rise to formidable computational challenges due to the vast amounts of data involved. In the human genome, for example, the problem of identifying genes in DNA sequences is well-known [1]. Furthermore, the complexity of many biological processes is such that the use of $a b$ initio methods is severely limited and so data mining techniques and statistical modelling must be used. While many of the techniques of informatics are well suited in this area the computing demands are so large that new models of computation, such as DNA computing, have been proposed. In this work we investigate possible implications of quantum computing in the areas of medical informatics and bioinformatics.

Through advances in both experimental technology and theoretical algorithm design [2] the research field of quantum computing has risen to prominence in the last decade. The idea behind quantum computing [3] is a fundamentally new type of computation based on the principles of quantum rather than 
classical physics. A consequence of this is that it is necessary to develop completely new types of computer architecture and this in turn requires the use of appropriate experimental techniques. Although the actual construction of a quantum computer is still some way off, a number of techniques have been proposed including the use of ion traps [4], NMR technology [5], cavity quantum electrodynamics [6] and solid state physics [7].

There are a number of reasons for investigating the relevance of quantum computing to informatics. First, as noted above the computational demands are such that qualitatively new types of computing need to be considered. Second, present trends in computer architecture suggest that quantum effects will become important within the next 20 years and so it will be essential to take account of these quantum phenomena even in the absence of quantum computing. Third, new quantum search algorithms [8], indicating that a quantum computer could outperform its classical counterpart, could have important applications in bioinformatics [9]. Finally, it is noted that quantum computers will be able to generate genuinely random numbers, which may be essential for simulating the dynamics of complex systems.

In this poster we give an overview of quantum computing and related areas such as quantum cryptography, which has already been demonstrated in realistic environments. We also investigate possible implications of quantum computing for research in the areas of data mining, and modelling and simulation.

\section{References}

[1] "Special Theme: Bioinformatics and Biocomputing” ERCIM NEWS 43 (2000)

[2] Shor P "Algorithms for Quantum Computation: Discrete Logarithms and Factoring" Proceedings of the 35 ${ }^{\text {th }}$ Annual Symposium on Foundations of Computer Science 124-34 (1994)

[3] Nielsen M and Chuang I Quantum Computation and Quantum Information

[4] Cambridge University Press (2000)

[5] Cirac J and Zoller P "Quantum Computations with Cold Trapped Ions" Physical Review Letters 74 4091-4094 (1995)

[6] Gershenfeld N and Chaung I "Quantum Computing with Molecules" Scientific American June 66-71 (1998)

[7] Turchette Q, Hood C, Lange W, Mabuchi H and Kimble H "Measurement of Conditional Phase Shifts for Quantum Logic" Physical Review Letters $\mathbf{7 5}$ 4710-4713 (1995)

[8] Kane B “A Silicon-Based Nuclear Spin Quantum Computer” Nature 393 133-137 (1998)

[9] Grover L "A Fast Quantum-Mechanical Algorithm of Database Search" Proceedings of the $28^{\text {th }}$ Annual ACM Symposium on the Theory of Computing 212-219 (1996)

[10] Hollenberg L C L "Fast Quantum Search Algorithms in Protein Sequence Comparisons: Quantum Bioinformatics" Physical Review B 62 7532-6 (2000)

\section{Optimization of the thermal protective clothing using a knowledge bank concept and a learning expert system}

L. Schwenzfeier ${ }^{\mathrm{a}}$, B. Warme-Janville ${ }^{\mathrm{b}}$, G. Delhomme ${ }^{\mathrm{a}}$, A. Dittmar ${ }^{\mathrm{a}}$

${ }^{\mathrm{a}}$ Biomedical Microsensors Microsystems, LPM-CNRS, UMR 5511, INSA de Lyon, Bât Leonardo da Vinci, 20 av. A Einstein, F - 69621 Villeurbanne Cedex, France

${ }^{\mathrm{b}}$ Centre d'Etudes du Bouchet, BPn 3 , F - 91710 Vert le Petit, France

Introduction: Protective clothing, required under hostile environment, reduces the man's capabilities because of heat stress. Protection materials have a very low vapour permeability and it decreases the evaporation capability, which is a natural cooling system of the human body. Moreover, the response of the nervous system to the mental stress caused by the clothes (especially the mask and respiratory system) is a vasoconstriction, which increase the core temperature.

The choice of the adapted protection for men and its optimisation, has to be taken into account to assume the task in its total duration and components. 
Measured parameters: The interactions man/clothing/environment are very complex and we have to take into account all the parameters that are relevant in the heat and mass balance: the human body and its several controlled systems, the multilayer protection, the interlayer, between skin and cloths, the environment and external conditions (fig. 1).

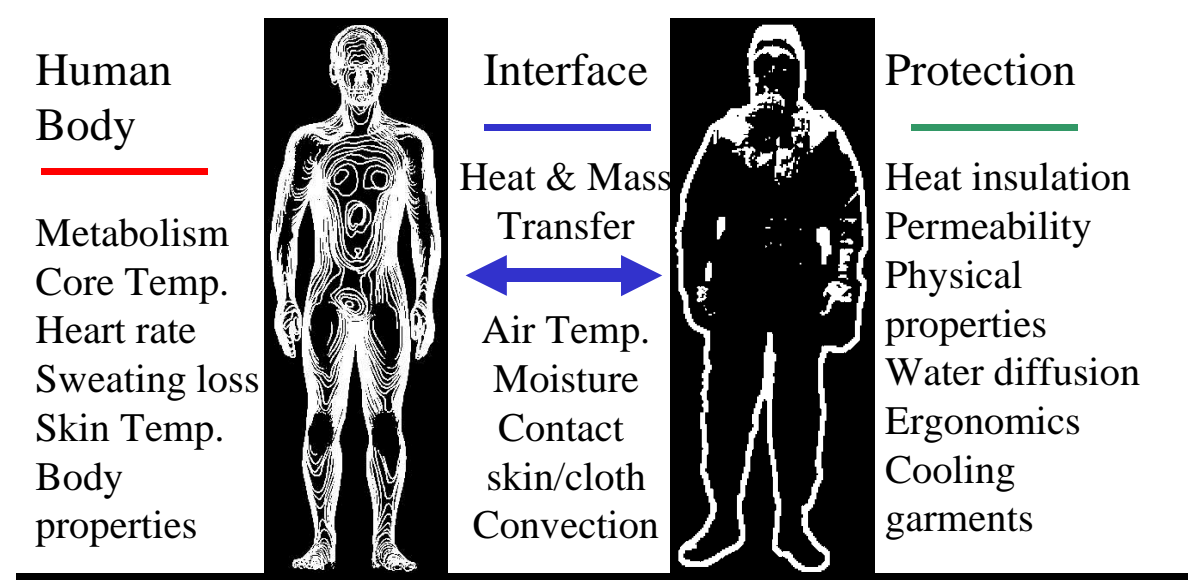

External conditions : Tasks, Ambient temp., Humidity, Wind, Duration, Equipment, ...

Fig. 1: Parameters involved in the heat and mass transfer man/clothing/environment.

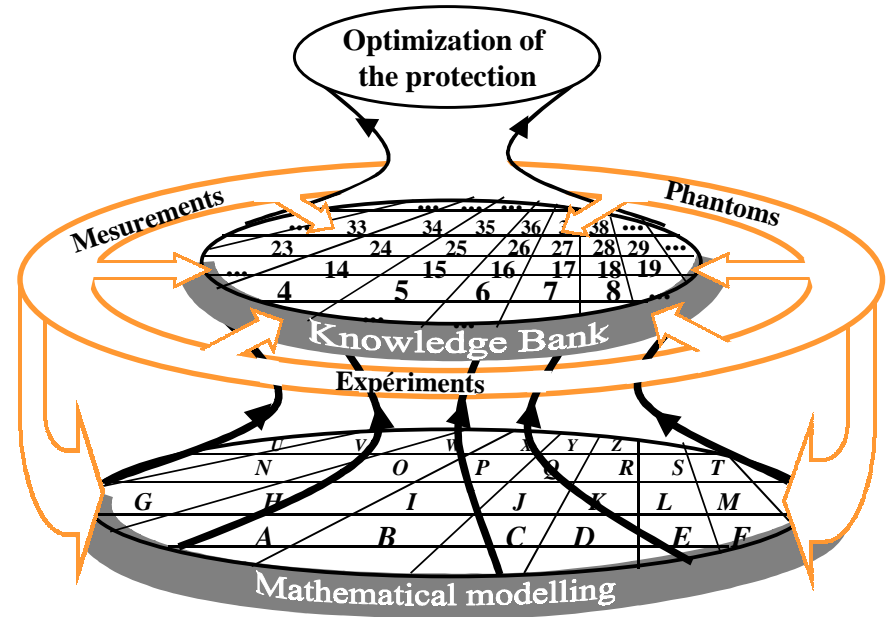

Fig 2: The Knowledge bank : Based on mathematical models, the synthesis of a set of scenarios allows the optimisation of the human protection (adapted protective clothing, minimum heat stress,...).

There are more than 150 thermal parameters (dimensional and dimensionless) acting in the human thermal system [1], [2]. The difficulty is to bring to the fore the relevant parameters. To study living tissues, we have to take into account: physical activity, heart rate, skin temperature, body properties (thermal conductivity, effusivity, emissivity,...), and of course sweating loss (and dehydration).

Protective clothing is multilayered, and composed by cooling garments. Water vapour permeability (usually very low), water diffusion, water absorption depend on the fabric fibers. These properties affect 
the sweat evaporation rate and also take a large part in the mass transfer. Thermal insulation of complete clothing, thermal resistance of the fabrics, effusivity, emissivity are also acting in the heat transfers.

However, the study of full garments requires the evaluation of more complex construction factors like design, cut, size, drape, complements (mask, gloves, etc).

Knowledge Bank: The man protection is necessary in numerous conditions and scenarios, and should not affect the work. Under hot and humid environment the principal problem is the raise of core temperature, which limits the operational capacities of the wearer in terms of skill and duration of the task.

Cooling garments and protective clothing used for military (aerospace, NBC suits, navy,) or industrial tasks (asbestos stripping, steal industry,...). Actually, modelling or using experimental procedure only isn't sufficient to optimise the protection for all requirement. The "knowledge bank" offers the possibility to integrate the existing models in their own field of application, and the results of experimentation on human subjects and phantoms.

The "knowledge bank" consists on a set of scenarios, integrating tasks requirements, external conditions, protective garment, physical activity allows and time course. This knowledge bank is "feed" by experiments and use all the modelling that exist on this field to synthesise results, useful to optimise the man protection.

Conclusion: While working under hostile environment, the most important consideration is the man's comfort and capabilities. But this is the conclusion of a very complex system that must be approached through several levels. Knowledge Bank is a synthetic, realistic, prediction tool, which gives the possibility to optimise the protection in different cases, numerous scenarios and situations. Dimensions of the different levels are not fixed. This method can integrate the new models, new scenarios, new experiments and also can fit to the future reality.

References

[1] J.C. Chato, A. Shitzer: On the dimensionless parameters associated with the heat transport within living tissue, Aerosp. Med. 41, (1970) p383-390.

[2] L.T. Fan et al.: A review on mathematical models of the human thermal system, IEEE Trans. Bio. Med. Eng., (1971) Vol BME 18, p218-234.

[3] L. Schwenzfeier, G. Delhomme, A. Dittmar : Effective insulation of protective clothing : a multiparametric approach. Congrès DGA-SFT-IIR. Thermal protection of man under hot and hazardous environment, pp. 343-348, Mars 99, Paris

\title{
Image display management for telecare applications
}

\author{
Mohsen Farid ${ }^{\mathrm{a}, \mathrm{b}}$ and Fionn Murtagh \\ ${ }^{a}$ Dundalk Institute of Technology, Dundalk, Ireland \\ 'Queen's University of Belfast, Belfast, Northern Ireland
}

Based on new image compression methods, we explore a number of image storage and delivery management systems. Compression is based on (i) image noise modelling, and (ii) wavelet or other multiresolution transforms. An example of a non-wavelet multiresolution transform which we have found to be very effective is the pyramidal median transform. Compression of either greyscale or colour images is supported. One in twenty compression rates are common, sometimes even for the lossless case.

By "delivery" we mean catering for the user's needs in terms of spatial resolution and region of interest. Full resolution display of a very large image is not wanted on typical display devices. Instead a lower resolution version of the image data permits global interpretation of the image information. Local 
interpretation is permitted by "zooming in" on a region of interest. Our compression storage formats are based on (i) resolution scale, and (ii) block regions, and hence support the desired delivery functionality.

Our demonstrators and prototypes (see reference) are based either on web servers, or as stand-alone systems for use from $\mathrm{CD}$ or on portable computers. For the latter area, graphical user interfaces have been built. For web-based systems, CGI-based implementations have been used.

We will describe the design and implementation of such multiresolution-based image management systems, and discuss a number of examples.

\author{
Reference \\ [1] "Multiresolution for Mobile Multimedia Applications", demonstrators and examples, http://www.multiresolution.com/mr- \\ mobile.htm
}

\title{
The learning and teaching support network - Promoting innovation and good practice in health and medical informatics
}

Sylvia Alexander ${ }^{\mathrm{a}}$, Margaret Sills ${ }^{\mathrm{b}}$ and Megan Quentin-Baxter

${ }^{a}$ Faculty of Informatics, University of Ulster, Shore Road, Newtownabbey, BT37 OQB, N. Ireland

${ }^{b}$ Franklin-Wilkins Building, King's College London, 150 Stamford Street, London SE1 8WA, UK

${ }^{c}$ Faculty of Medicine, University of Newcastle, Newcastle upon Tyne NE2 4HH, UK

The UK higher education funding bodies have established a Learning and Teaching Support Network (LTSN) to promote high quality learning and teaching in all subject disciplines in higher education. The network, which consists of 24 subject centres together with a generic learning and teaching centre, supports the development and transfer of innovation and good practice by providing a 'one-stop-shop' for the exchange of knowledge and expertise relating to teaching, learning and assessment.

The discipline focus of this programme recognises that for many in HE most networking and exchange of practice and innovation takes place at the subject level. Subject centres are also mindful of the need to address emergent interdisciplinary topics, including health and medical informatics, which are not highlighted under the remit of any of the Subject Centres.

Health and medical informatics (HMI) is an emerging multidisciplinary topic at the interface between information technology and the disciplines of medicine and health care. HMI appears on the curriculum of a number of undergraduate courses including biomedical engineering and medicine and a growing number of advanced postgraduate and professional development programs are being offered. Students of HMI come from diverse academic backgrounds including informatics, engineering, medicine and health care professionals. In recognition of this fact the LTSN Subject Centres for

- Information and Computer Science (based at the University of Ulster)

- Health Science and Practice (based at King's College, University of London) and

- Medicine, Dentistry and Veterinary Medicine (based at Newcastle University)

will collectively provide a service to support learning and teaching in this important and rapidly developing area.

Advances in information and communication technologies have already enhanced the quality of health care bringing significant economic benefits. If health care professionals and medical practitioners are to exploit these new technologies to their full potential then high quality education in this area is required. The LTSN Subject Centres will promote the integration of appropriate communication and information technology techniques to enhance learning and teaching. 
The Centres deliver both a proactive and responsive service to their respective discipline communities and aim to encourage active participation from the widest possible audience. Amongst the services offered are

- Comprehensive websites providing immediate access to information and resources to support teaching and learning

- An extensive programme of events (including web-based events) providing opportunities for the exchange of information and enabling participation and interaction throughout the discipline communities

- Paper and electronic publications including evaluations and case studies of good practice

- An advice service to provide support for individuals and departments

- Electronic mailing lists and focus groups to support specialist networks for exchange and dissemination of expertise within the disciplines (including health and medical informatics)

- Regular news services to keep the community informed of activities within the LTSN network and of pedagogical and technological innovations relevant to discipline specialists.

Further information on the Learning and Teaching Support Network and the relevant Subject Centres is available via the websites at:

LTSN: http://www.ltsn.ac.uk

LTSN Subject Centre for Information and Computer Sciences: http://www.ics.ltsn.ac.uk

LTSN Subject Centre for Health Science and Practice: http://www.health.ltsn.ac.uk/

LTSN Subject Centre for Medicine, Dentistry and Veterinary Medicine: http://www.ltsn-01.ac.uk/

\section{A neural network prediction model to assist in the design of myocardial infarction classifiers}

C.D. Nugent, J.A. Lopez, A.E. Smith, N.D. Black

Medical Informatics, Faculty of Informatics, University of Ulster at Jordanstown, N. Ireland

Abstract: The design of Neural Network (NN) classifiers, which address the problem of electrocardiogram (ECG) classification, have inherent heuristic design procedures. It has been the aim of this study to generate prediction models to indicate the point at which training should cease to ensure maximal generalisation based on 5 variable network design parameters. The prediction models, based on NNs, were evaluated on three different data sets of Myocardial Infarction (MI). The results from both training and testing indicated no significant differences between actual and desired outputs $(p>0.05)$.

Introduction: The use of NNs in the classification of the ECG has proven, in recent years, to be a popular and successful choice [1]. A problem, however, with the use of such classifiers are their heuristic design procedures. During the design of a NN, such as a multi-layered perceptron (MLP), issues of their architecture, how many hidden layers should be included, how many nodes should each layer have etc. must all be addressed. In addition to these issues, the point at which the network is considered to be acceptably trained must be located.

During the training of a network it can be observed that the learning performance increases monotonically. By monitoring the performance of the $\mathrm{NN}$ on unseen data throughout training, it can be seen that the performance increases monotonically to a maximum, then decreases gradually as training continues. The suggested point of stopping training is considered to be at the maximum point of performance attained during testing [2]. It has been the aim of this study to develop prediction models to 


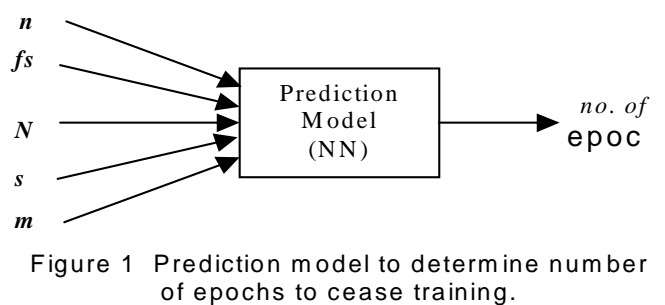

indicate the point at which training should cease, i.e. maximum test performance, based on the design parameters associated with a given network.

Methods: The data for the current study has been generated from a previous study undertaken by the authors whereby a classification framework for diagnosing the ECG was established based on a framework of NNs [3]. The variable design parameters for each NN developed included the number of nodes in the hidden layer (n), feature selection method (fs), number of files in the training set $(\mathrm{N})$, the size of the input feature vector (s) and the point at which the network attained maximum performance during training (number of epochs) (m). The data for NNs generated for Inferior MI (IMI), Anterior MI (AMI) and combined MI (CMI) were analysed. Figure 1 represents an overview of the NN prediction model developed to indicate the point at which training should cease.

Results: For each pathology an MLP NN with one hidden layer was developed with the aforementioned variable criteria as inputs. Each network had one output node, indicating the epoch number at which training should cease to ensure maximal generalisation. Table 1 shows the results for

\begin{tabular}{|l|c|c|c|c|c|}
\hline \multicolumn{1}{|c|}{ NN } & $\begin{array}{c}\text { Number of } \\
\text { cases }\end{array}$ & $\begin{array}{c}\text { Mean } \\
\text { Rank -ve }\end{array}$ & $\begin{array}{c}\text { Mean } \\
\text { Rank +ve }\end{array}$ & z-value & $\begin{array}{c}\text { 2-tailed } \\
\text { sig }\end{array}$ \\
\hline AMI Train & 29 & 17.73 & 13.33 & -0.487 & $\mathrm{p}=0.627$ \\
\hline AMI Test & 15 & 7.00 & 8.67 & -1.023 & $\mathrm{p}=0.306$ \\
\hline IMI Train & 31 & 16.73 & 15.31 & -0.059 & $\mathrm{p}=0.953$ \\
\hline IMI Test & 15 & 8.33 & 7.78 & -0.568 & $\mathrm{p}=0.570$ \\
\hline CMI Train & 37 & 16.30 & 23.43 & -0.355 & $\mathrm{p}=0.723$ \\
\hline CMI Test & 14 & 6.80 & 7.89 & -1.161 & $\mathrm{p}=0.245$ \\
\hline
\end{tabular}

Table 1 Results following evaluation for predictions models.

each prediction model following comparison with the actual network output and the desired output. As can be seen, the results indicate that there are no significant differences ( $>0.05)$ with respect to predicted and actual epoch values.

Conclusions: In the present study, based on 5 variable parameters of a network's design, it has been possible to predict when training should terminate to ensure maximal generalisation. For the three pathologies investigated, no significant differences between the actual and desired epoch values were recorded. This is a powerful result and assists in alleviating the amount of work to be undertaken in the design of a NN classifier.

The results from this study indicate that it is possible to generate a prediction model to deduce the point at which training should cease for NN ECG classifiers. 
References

[1] Bortolan, G, Brohet, C. and Fusaro, S. Possibilities of using neural networks for ecg classification. Journal of Electrocardiology, vol. 29, pp 10-16, 1996.

[2] Combining Artificial Neural Nets: Ensemble and Modular Multi-Net Systems, Sharkey, A.J.C. (ed.), Springer-Verlag, London, 1999.

[3] 3. Nugent, C.D., Webb, J.A.C, Black, N.D., Wright, G.T.H. and McIntyre, M., An intelligent framework for the classification of the 12-lead ecg, Artificial Intelligence in Medicine, vol. 16, pp. 205-222, 1999.

\title{
Barriers to evidence based practice - The dependence on medical informatics
}

\author{
W.G. Kernohan \\ Institute of Postgraduate Medical and Health Sciences, University of Ulster, Northern Ireland
}

Introduction: Evidence-based practice is the latest bandwagon in healthcare yet arguably the route to the best possible practice. Increasingly evidence plays a greater role in the decisions undertaken by all healthcare professionals.

With the aim of improving clinical practice, the objectives of this paper were:

- to review the capacity to implement evidence-based practice amongst health workers,

- to audit the extent of evidence-based practice,

- to identify barriers to progress.

Methods: 850 Nurses, health visitors and midwives were identified from human resource listings, by grade and sent a 20-item questionnaire covering basic demographic details of work place, current grade, qualification year and highest qualification obtained; experience of research; computer access and experience; and journal access and use. The questionnaire also elicited perceived extent of evidencebased practice and agreement with various barriers: cost; time; ease of implementation; training need; need for librarian; colleagues; position in organisation; perceived reward and opportunity for personal development. Descriptive and comparative analysis was performed for each site and an overall average value calculated for all variables.

Results: Staff returned a total of 288 questionnaires: a response rate of approximately one third. Responders represented the great majority of Trust service-delivery sites: 15 from a total of 16 . Each response was in proportion to the numbers employed at each place with a wide variety of numbers responding (range 0-78). Staff qualified mainly in the two decades 1970-1989 with a professional Diploma or Certificate. Graduate staff are in the minority with the average site proportion being 14\% graduate. Taken as a group, staff had barely any research experience.

Computer availability is an important component of the exercise of evidence-based practice. Around $25 \%$ of staff cite no access to computers, some $15 \%$ claim access in a local library; $21 \%$ have computer access at work; and importantly $48 \%$ have access at home.

Around 30\% have no experience of computers with one third having recreational use, one third having had some experience as a student and just $11 \%$ describing their computer experience as "professional"; by implication an important proportion has no experience of the use of computer in their profession.

The average site regarded $62 \%$ of practice to be based on evidence and generally staff perceive much of their practice to be evidence-based. However, approximately half (48\%) recognise cost of implementation as a major problem. Few (31\%) have sufficient time to spend putting research into practice. A similar proportion (37\%) found it easy to put research into practice. Many (62\%) see their training needs as a potential barrier to evidence-based practice. 
When posed with the possibility that "I would be more confident if there was someone allocated to give help finding information" most staff replied that they would be more confident if a librarian or informaticist was allocated to help with evidence seeking. Most (average 92\%) would see their colleagues as supportive in putting evidence into practice. Only a small number of staff agreed with the proposition that "there are few benefits to changing the current practice". They largely recognise their own position to influence changes in clinical practice.

Discussion: Evidence based healthcare has the potential to deliver best practice and meet the needs of clinical governance. However, due to the pervasive need to base actions and decisions upon evidence it cannot be conveniently bolted-on or bought-in from outside. It is a key quality issue requiring the development of key skills, resources and support. Medical Informatics is at the core of this activity. It requires to be taught, learnt, practised and supported.

Barriers to evidence based practice include a lack of knowledge, experience and interest; difficulty with nature, cause and conceptualisation of the problems in the delivery of health care; problems with inter-disciplinary relationships and teamwork; problems with a lack of autonomy in decision making; and problems with personal and organisational management.

There is a widespread lack of supporting infrastructure; inability to access suitable IT; inertia, particularly in primary care; lack of guideline ownership and difficulty handling the volume of evidence. Lack of time for evidence based practice is often cited as the main problem.

Research and development in health services have been recognised as being fragmented; lacking a clear strategy and vision; and isolated from the wider body of research in the health arena. Five key issues have been identified: setting research priorities; increasing the influence of staff on the ground upon the wider R\&D Agenda; attending to the training and support needs; and improving the dissemination and uptake of research findings.

This research supports much of what has been said about evidence-based practice in terms of facilitation, resources and training.

\section{Home control system for the social integration of disabled people}

Jose A. Vera, Joaquin Roca and Manuel Jiménez

Industrial \& Medical Electronics Research Group (EIMED) Joaquin.Roca@ upct.es

Electronic Technology Department - Polytechnic University of Cartagena, Spain

Introduction: In recent years many systems have been developed to allow the integration of persons with disabilities (PWDs), particularly those with severe brain disease, which in many cases are caused by severe brain damage. Psychologists and Medical specialists have come to the conclusion that the psychological stimuli induced in the PWDs by actions which provide them with autonomy at home, improve the rehabilitation and the achievement of new mental and physical capabilities.

All these approaches have provided valid solutions, but with the important drawback of not being based on commercial, flexible and integrated systems. This has led to the inability to unify efforts from different research centers specialized in this subject.

Our main objective in this project has been to design and develop a home control system accessible to both people with different degrees of disability and persons with all their capabilities; the so-called "Design For All”.

The proposed solution has taken advantage of the high flexibility of the EIB system [1,2,3], as well as the numerous tools available for the development of new applications. 
System Requirements: Our system should allow the following actions:

- Safety and security

- Home Control: light, temperature, shutters, access control (audio + video).

- Communication: telephone calls, emergency calls, etc.

The System Structure: Figure 1 shows the different elements integrated in our system. A conventional EIB system has been used to provide access to the typical home components (sensors, switches, etc), what give us the key feature to integration: the possibility of control through a personal computer, which would be used to interface with PWDs.

This led us to propose two different solutions: on one hand we may implement in this computer the access interface for PWDs (based on raster-scan techniques) and the software for bus control, on the other

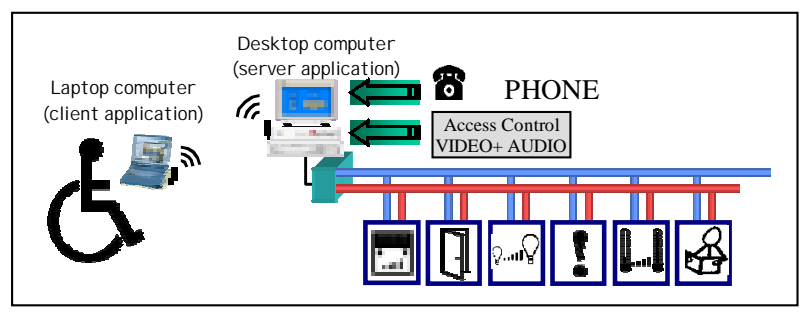

Fig. 1 System Structure

hand the application for bus control can be programmed on a desktop computer, and the interface for PWDs on a laptop.
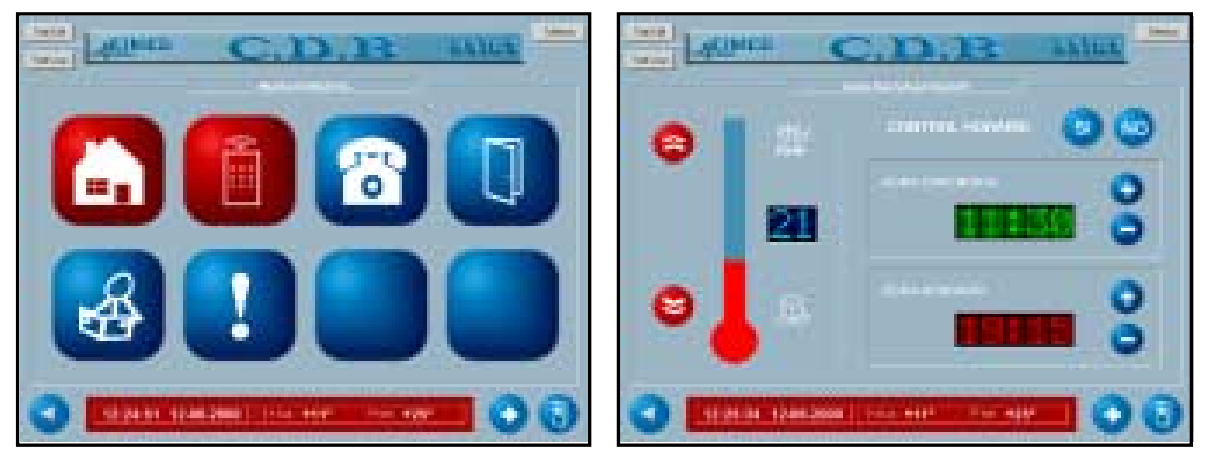

Fig. 2 Custom Graphical User Interface

This implementation increases the flexibility of the global system, as it provides a standard, high-speed communication link (from 2 to $11 \mathrm{Mbps}$ ) and full mobility for the PWD.

Both the client and server applications have been developed in $\mathrm{C}++$. The first (running in the laptop computer), is an amiable graphical interface (figure 2) suitable for any kind of user (PWD or not).

For this purpose, the interface has the possibility of being operated using either raster-scan techniques or a conventional mouse or tactile display. Its functionality covers a wide range of house components in every room of the house. 
Results: This system has proved itself as efficient to stimulate the PWDs, which significantly increased their autonomy.

Discussion: The system could be improved in the design of new GUIs specially conceived for each kind of disability (i.e. by including a speech recognition engine as input processor, etc.) without loosing the guidelines of the "Design For All".

The most important efforts related to Biomedical Engineering fields should lead to the integration of a remote-care and remote-diagnostic system.

\title{
References
}

[1] Various "Project Engineering for EIB installations \& Applications" EIBA 1998.

[2] European Bus Association. http://www.eiba.com

[3] Remartinez, Antonio. "Aplicaciones para mejorar la calidad de vida: Domótica- teleasistencia y control de entorno" Novática Julio 2000

\section{Posters for Bioengineering}

\section{A comparative study of three techniques for QRS cancellation in the ECG}

\author{
M. A. Diaz, O. Escalona, A. I. Hernández, F. Mora \\ Grupo de Bioingeniería y Biofísica Aplicada (GBBA), Universidad Simón Bolívar, Apdo. 89000, Caracas \\ 1080-A, Venezuela \\ E-mail:mdiaz@gbba.usb.ve
}

Introduction: Most $\mathrm{P}$ wave detection methods reported in the literature to date, are based on the cancellation of the QRS complex and the T wave in the ECG, followed by nonlinear transformations applied to the residual signal as the final step in the detection procedure. Other proposed schemes have used adaptive filtering techniques [1-2] and neural network based techniques [3].

Here, the performance of three QRS cancellation schemes were compared. These techniques are used in order to obtain a residual ECG which contains only atrial activity information, thus facilitating P-wave detection in a later stage and in the analysis of atrial arrhythmias. The considered schemes are based on: a) multiband (MB) and adaptive filtering [2], b) neural networks (NN) [3], and c) on the spatial-temporal alignment of an average beat which is subtracted from the original ECG signal [4]. The adaptive filter scheme proposed by Thakor was used as the reference for comparison.

Methods: All schemes were tested using records number 100, 108 and 222 of the MIT-BIH ECG database. Base line wandering removal was applied prior to processing. Quantitative assessment of the performance of the various schemes was implemented using new parameters introduced by Vásquez and Pérez [2-3], in where the beat to beat estimated energy of the atrial activity $\left(\mathrm{E}_{\mathrm{AA}}\right)$ and of the ventricular activity $\left(\mathrm{E}_{\mathrm{VA}}\right)$ of the residual signals resulting after applying a particular cancellation method, are used as the performance indicators. Figure 1 shows a block diagram of the implemented Spatio-Temporal Alignment (STA) cancellation technique [4]. 


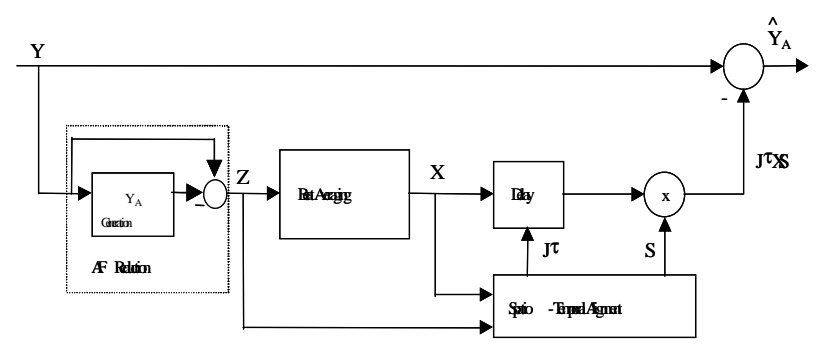

Fig. 1. Spatio-Temporal cancellation of QRST.

Results: Table I shows the results of the calculated median of $\mathrm{E}_{\mathrm{AA}}$ before and after cancellation of the ventricular activity, here record 222 was not included because it does not provide the notation of $\mathrm{P}$ wave activity, as in this record are many segments with atrial fibrillation. Table II shows the results related to $\mathrm{E}_{\mathrm{VA}}$, and Table III shows the calculated values of signal to noise ratios (SNR) in the various cancellation techniques.

Table 1. Median of $\mathrm{E}_{\mathrm{AA}}$.

\begin{tabular}{|c|c|c|c|c|c|c|c|}
\hline \multirow[b]{3}{*}{ Record 100} & \multicolumn{7}{|c|}{ Cancellation Method } \\
\hline & $\underset{(\operatorname{Lead} \mathbf{I})}{\mathbf{E}_{\mathrm{AA}}}$ & $\begin{array}{c}\mathbf{E}_{\mathrm{AA}} \\
\text { (Lead } \\
\text { II) }\end{array}$ & $\begin{array}{c}\mathbf{E}_{\mathrm{AA}}(\mathrm{LI}) \\
\text { Thakor }\end{array}$ & $\begin{array}{l}\mathbf{E}_{\mathrm{AA}} \mathbf{N N} \\
\text { (Lead I) }\end{array}$ & $\begin{array}{r}\mathbf{E}_{\mathrm{AA}} \mathbf{M B} \\
\text { (Lead I) }\end{array}$ & $\begin{array}{c}\text { E }_{\text {AA }} \text { STA } \\
(\text { Lead I })\end{array}$ & $\begin{array}{c}\mathbf{E}_{\mathbf{A A}} \mathbf{S T A} \\
\text { (Lead } \\
\text { II) }\end{array}$ \\
\hline & 172.9458 & 37.5736 & 186.2696 & 86.2161 & 87.4814 & 172.9170 & 37.5736 \\
\hline Record 108 & 647.6132 & 160.2963 & 640.5408 & 641.1130 & 318.9369 & 647.2663 & 159.8288 \\
\hline
\end{tabular}

Table 2. Median of $E_{\mathrm{VA}}$.

\section{Cancellation Method}

\begin{tabular}{|c|c|c|c|c|c|}
\hline$\underset{(\text { Lead I) }}{\text { EVA }_{\text {VA }}}$ & $\begin{array}{c}\mathbf{E}_{\mathrm{VA}} \\
(\mathbf{L e a d} \text { II })\end{array}$ & $\begin{array}{c}\mathbf{E}_{\mathrm{VA}}(\mathbf{L} \mathbf{I}) \\
\text { Thakor }\end{array}$ & $\begin{array}{r}\text { EVA }_{\text {NN }} \\
\text { (Lead I) }\end{array}$ & $\begin{array}{l}\mathbf{E}_{\mathrm{VA}} \mathrm{MB} \\
\text { (Lead I) }\end{array}$ & $\begin{array}{c}\text { E }_{\text {VA }} \text { STA } \\
\text { (Lead I) }\end{array}$ \\
\hline
\end{tabular}

II)

\begin{tabular}{llllllll} 
Record 100 & $1.8829 * 10^{3}$ & 805.4406 & 83.3333 & 80.6661 & 59.8939 & 29.5737 & 27.4568 \\
\hline Registro 108 & $1.7825 * 10^{3}$ & $6.0840 * 10^{3}$ & 185.4285 & 150.8202 & 54.7410 & 115.1735 & 86.5368 \\
\hline Registro 222 & 758.0482 & $1.2699 * 10^{3}$ & 208.7554 & 196.8541 & 176.8206 & 158.0445 & 31.9154 \\
\hline
\end{tabular}


Table 3. Estimated SNR.

\begin{tabular}{lcccccc}
\hline & \multicolumn{5}{c}{ Cancellation Method } \\
& Thakor & NN & MB & STA LI & STA \\
& & & & & LII \\
Record 100 & 3.4484 & 0.3753 & 1.4710 & 7.6486 & 1.2738 \\
& & & & & \\
Record 108 & 5.6436 & 6.1106 & 9.8711 & 7.4083 & 3.0478 \\
\hline
\end{tabular}

Conclusions: The adaptive schemes based on neural network (NN) and multiband adaptive filtering (MB) are more efficient in the presence of morphological changes and noise in the signal than their counterpart (STA). Morever, the adaptive schemes have the relative advantage that they do not need a previous QRS detection stage. However, an improved performance was obtained with the spatiotemporal alignment scheme whenever there was no misdetections of QRS beats along the ECG recording.

\title{
References
}

[1] N. Thakor, Y. Zhu. Aplications of Adaptive Filtering to ECG Analysis: Noise Cancellation and Arrhytmia Detection. IEEE Transactions on Biomedical Engineering, 48:8, pp 785-794, 1991.

[2] B. Pérez. Filtraje Multibanda y Anulación Adaptativa del QRS para la Detección de la Onda P. Tesis de Maestria, Universidad Nacional Experimental Politécnica “Antonio José de Sucre”. 1999.

[3] C. Vásquez, A.I. Hernández, F. Mora, G. Carrault, G. Passariello. Feasibility of Neural Network

[4] M. Stridh, L. Sörnmo. Spatiotemporal QRST Cancellation Techniques for Improved Characterization of Atrial Fibrillation in the Surface ECG. Proceedings - 19th International Conference IEEE/EMBS, pp 48-49, Chicago, USA, 1997.

\section{Medical image based personalised tibial component: biomechanical evaluation}

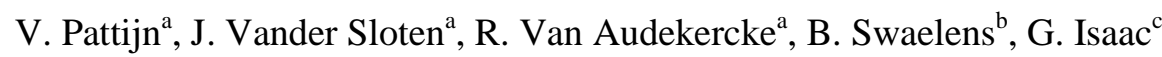 \\ ${ }^{a}$ Division of Biomechanics and Engineering Design, K.U.Leuven, Belgium \\ ${ }^{b}$ Materialise N.V., Belgium \\ ${ }^{c}$ DePuy International, $U K$
}

Introduction: One of the major causes of failure in total knee arthroplasty is loosening of the tibial component. This loosening phenomenon is mainly due to bone resorption or local overloading. It also depends on the quality of the underlying bone, the implant design and the implantation technique [1,2]. The implant design and the implantation technique can be improved towards the conditions specific for each individual patient, taking into account patient's bone geometry and material properties. In the absence of tibial component-cortex contact, the trabecular bone of the proximal tibia must transfer a higher load than in the intact joint; hence there is a risk of implant loosening and/or sinkage [3]. Therefore this study will look at the design and biomechanical evaluation of a personalised tibial component with a full component-cortex contact.

\section{Methods}

Design: First a CT-scan was made of a cadaver tibia. Based on these medical images a 3D-model of the tibia was created. On this 3D-model the surgeon has planned the resection of the proximal tibial joint surface (cutting plane), in a similar way to the placement of the tibial component in a total knee 
arthroplasty. A standard tibial component (AMK ${ }^{\circledR}$ tibial component from DePuy) with a central stem and two pegs was positioned as good as possible in these virtual images. Then the outer contour of the tibial component was adapted to the outer contour of the tibia in the resection plane. This resulted in a first custom tibial component that exactly fits on the outer contour of the tibia, hence fully supported by the cortical bone. Based on this first design two more tibial components were defined: one with a $1 \mathrm{~mm}$ inward offset of the outer contour and one with a $1 \mathrm{~mm}$ outward offset of the outer contour. The fourth design was a tibial component with a smooth (lower order) approximation of the exact contour of the tibia.

Biomechanical evaluation: We are interested in the effect of the cortical support of the tibial component upon cortical bone strains in the bone beneath the tibial component under different loading conditions. Therefore the following test protocol is performed.

First the main strains and directions in the cortex of the intact cadaver tibia were measured at 10 different positions with strain gauges (Measurements Group, Inc., CEA-06-125UR-120) under the following loading conditions:

1. compression load of $320 \mathrm{~N}$

2. compression load of $700 \mathrm{~N}$

3. compression load of $1400 \mathrm{~N}$ under $20^{\circ}$ of knee flexion.

Then the same experiments were performed on the tibia with one of the custom tibial components implanted. The custom implants were tested in the following order:

1. component with $1 \mathrm{~mm}$ outward offset $(\mathrm{CO})$

2. component with exact fit (CF)

3. component with smooth approximation of the outer contour (CS)

4. component with $1 \mathrm{~mm}$ inward offset (CI)

Results: The strains measured in the intact tibia show a different pattern than the strains measured in the tibia with a custom component implanted, and that for all three loading conditions. For the four custom designs a similar strain pattern is observed under the first two loading conditions. Only when simulating the load under $20^{\circ}$ knee flexion, there is a difference in strain pattern observed between the four components. $\mathrm{CO}$ and $\mathrm{CF}$ have a similar pattern and also CS and CI.

Discussion: From these experiments it appears that a full support of the tibial component by cortical bone, yields a similar strain distribution in the underlying cortical bone as in the case of a poor cortical coverage. Hence we can conclude that the load transfer from the component to the tibia is not mainly determined by the cortex but also by the distribution through the trabecular bone to the cortex. The strain pattern is totally different for the intact tibia than for the case with a tibial component implanted.

\section{Acknowledgement}

This research was performed in the Brite Euram project PISA (NR. BRPR CT97 0378).

References

[1] M.M. Petersen, P.T. Nielsen, A. Lebech, S. Toksvig-Larsen, B. Lund, J. Arthroplasty 14: 77-81 (1999).

[2] R.W. Lee, R.G. Volz, D.C. Sheridan, Clin. Orthop. 273: 177-183 (1991).

[3] R.B. Bourne, J.B. Finlay, Clin. Orthop. 208: 95-99 (1986). 


\section{Posters for Medical Instrumentation \& Imaging}

The design of a compact, multi-element test object for use in Monte Carlo calculations in diagnostic radiology

J. Ning ${ }^{\mathrm{a}}$, K. Cranely ${ }^{\mathrm{b}}$, S.McClean ${ }^{\mathrm{a}}$

${ }^{a}$ Faculty of Informatics, University of Ulster, N. Ireland, UK

${ }^{\mathrm{b}}$ Radiation Protection and Imaging Section, Northern Ireland Regional Medical Physics Agency, UK

Introduction: In the past, television fluoroscopic systems in hospitals were not necessarily set up correctly by their manufacturers due to the many forms and wide range of performance. In this context, test objects were first designed by Hay and Clarke ${ }^{1}$ to control the image quality. Today, the general design of X-ray fluoroscopic systems has been largely, but not completely standardised. However, test objects are still necessary since a system that is exemplary in the factory and in the laboratory may be grossly maladjusted in the field.

Monte Carlo simulation is a well-known method for modelling radiation fields. It has been applied to diagnostic radiology for image analysis and for patient radiation dosimetry, but except for quite limited applications, not for both of these at the same time. In our research, a Monte Carlo code MCNP4B has been used. It allows the x-ray beam/patient/image receptor system to be modelled, so that various parameters can be varied allowing the simultaneous effect of these changes on both image quality and patient dose to be investigated. Many of these simulations are estimated by assuming a homogeneous phantom representing the patient ${ }^{2}$. This restrictive assumption can be realised by a voxel phantom known as NORMAN ${ }^{3}$, in order to improve the precision of the estimation.

In using Monte Carlo calculations for imaging purposes, images comparable in quality to clinical images obtained using image intensifier or film-screen combinations, require extremely large numbers of photons to be generated. For example, to generate the equivalent of one second of fluoroscopic screening at $1 \mathrm{~mA}(70 \mathrm{kV}$ at $1 \mathrm{~m})$, the Monte Carlo program would need to run, typically, for 2 hours on a $266 \mathrm{MHz}$ computer, for each $\mathrm{cm}^{2}$ of image produced, referred to a homogeneous $200 \mathrm{~mm}$ thick PMMA phantom. It is therefore necessary to be as economical with the photons generated as possible in the respect of Monte Carlo simulation.

The test object described here is designed to make optimum use of the available x-ray beam, by minimising the area irradiated but not used. The x-ray beam has a conical shape with a circular crosssection. Progressively increasing the radius of the circular cross-section permits the inclusion of more features of the test object, up to a maximum radius of approximately $70 \mathrm{~mm}$. Similarly, by selecting an annular cross-section with inner and outer radii, various combinations of image quality tests can be obtained.

Description of the Test Object: The test object is designed based on the presumption that it is necessary to be as economical with the photons generated as possible. It has a circular shape and four regions. The inner section consists of a star pattern with $2^{\circ}$ wide, $0.1 \mathrm{~mm} \mathrm{~Pb}$ thick, spokes at $2^{\circ}$ intervals. This test allows an indirect measurement of the dimensions of the x-ray tube focal spot. Region 2 consists of a concentric annulus, divided into four equal segments - one which allows a 'background' signal to be recorded on the image, and three 'shadow' regions, containing $3 \mathrm{mgcm}^{-2}, 6 \mathrm{mgcm}^{-2}$ and $12 \mathrm{mgcm}^{-2}$ of iodine. This allows measurement of signal-to-noise ratio by determining the energy absorbed and the corresponding statistical variation of the 3 regions of the image receptor corresponding to the 'shadow' regions. Region 3 is a copper step wedge with $0.1 \mathrm{~mm}$ copper steps from 0 to $3.1 \mathrm{~mm}$ copper, of concentric annular segment shape which can be used in conjunction with a scattering medium. This 
object allows the relationship between image optical density and entrance air kerma to be determined, ie the 'characteristic curve' of an image receptor such as a film-screen combination. In the case of a Monte Carlo simulation, the measured characteristic curve from a real image receptor can be used to modify the image densities calculated. Region 4 contains twelve sets of contrast-detail objects. Each set comprises nine similarly-size circular discs, which have a range of radiographic contrasts. Each of the 12 sets has a different size of circular discs. When imaged with x-rays, the observer can visualise some, but not all of the discs of each size. This subjective test allows a Contrast-Detail Diagram to be constructed.

Methods: The geometry of the simulation includes a $0.6 \times 0.6 \mathrm{~mm}$ rectangular x-ray tube focal spot, projecting a conical $x$-ray beam downwards with its vertical central axis corresponding to that of a $250 \times$ $250 \times 200 \mathrm{~mm}$ deep PMMA phantom. The test object is embedded in this PMMA phantom. Within the phantom-to-image-intensifier gap is an air-filled ionisation chamber with negligible wall thickness, positioned between the fibre grid (above) and an Al image intensifier cover (below). This ionisation chamber is used to monitor the image intensifier input air kerma. The CsI image intensifier is divided into regions of interest which are used for monitoring the radiation projected through each of the concentric annular segments of Region 2 and 3. This is necessary for determining the energy deposited in the image intensifier and its statistical variation corresponding to the areas of Region 2 for SNR calculations. The energy deposited in each region of interest of the image receptor corresponding to each step of the $\mathrm{Cu}$ step wedge is required for use with the characteristic curve.

Summary: The test object contains two subjective and two objective tests. The objective tests include a step-wedge for the image receptor characteristic curve and a signal-to-noise ratio $(S N R)$ test. The $S N R$ test allows a comparison between image quality (defined crudely as output $S N R$ ) and the phantom/patient average dose $D$. The parameter 'dose-to-information conversion efficiency' given by $S N R^{2} / D$ is proving to be useful for optimising imaging systems, but because this is a first-order measure, the current research examines the effects of this optimisation on other more detailed aspects of image quality. The two subjective tests are Regions 1 and 4, i.e. the star pattern and the contrast-detail test. These test both result in measurements which are reproducible, provided an experienced observer carries out the evaluation.

\title{
References
}

[1] G.A.Hay, O.F.Clarke, N.J.Coleman, and A.R.Cowen, A set of X-ray test objects for quality control in television fluoroscopy, The British Journal of Radiology, Vol. 58, 335-344, April 1985.

[2] Markku J Tapiovaara, Michael Sandborg and David R Dance, A search for improved technique factors in paediatric fluoroscopy, Phys. Med. Bio. Vol.44, 537-559, 1999.

[3] Dimbylow PJ, The development of realistic voxel phantoms for electromagnetic field dosimetry, Proceedings of an Interantional Workshop 'Voxel Phantom Development', 6 \& 7 July,1995.

\section{Medical engineering technologies for diagnosis and correction of higher mental function in children and adolescents}

\author{
V.A. Viktorov, G.I. Kavalerov, E.V. Matveev \\ Medical Engineering Research Institute (JSC «VNIIMP-VITA»), Russian Academy for Medical Science, \\ Moscow, Russia
}

Introduction: The subject of present work is of considerable theoretical and practical importance. A large number of global and local factors (ecological, social, economical, intellectual, emotional, etc.) exert a significant impact on the human nervous system. Children and adolescents are the most vulnerable to detrimental factors. Development disorders affect new generations of our society. The problem of ade- 
quate and multilateral development of new healthy generations is of cardinal and global social importance.

The goal of researchers is to develop new medical engineering technologies designed to provide prophylaxis, diagnosis, therapy, and rehabilitation of psychoneurological diseases.

The goal of this work was to describe new medical devices and systems designed at VNIIMP-VITA, Ltd. in collaboration with other organizations for the purpose of quantitative psychophysiological testing and rehabilitation of higher mental activity of children and adolescents in normalcy and pathology.

The theory of functional systems, medical cybernetics, neuropsychol-ogy, and systemic psychology (P. K. Anokhin, A. 1. Berg, A. R. Luriya, and B. F. Lornov) provided the theoretical basis for these studies.

Method and Design: Several generations of devices and systems for psychophysiological examinations have been developed and introduced into medical practice on this theoretical basis. The following systems have gained the most widespread recognition.

The PSYCHOMATH research system is designed to provide various modes of psychophysiological examination (fig. 1). This system is based on an integral microcomputer and a large body of software. The PSYCHOMATH system implements some routine psychophysiological methods and various psychodiagnostic questionnaires. The combined use of instrumental psychophysiological methods and psychological tests significantly expands the range of functional performance of the device.

The RITHMOTEST, MNEMOTEST and BINATEST devices were developed based on conventional methods of psycho-physiological examinations. These devices are designed for testing specific cognitive functions. The RITHMOTEST device is used for testing psychomotor arrhythmia; the MNEMOTEST device, for testing visual perception and visual memory using special test patterns; and the BINATEST device, for testing target-oriented behavior, decision-making processes, and probabilistic prognosis in the central nervous system. This device implements various measuring modes, including estimation of decision stereotype, effect of reward, adaptability, and specificity of sequential behavioral events in stochastic and deterministic media of various structure. The Ataksitest device is designed for testing ocular, motor, and hand tremor as well as static and dynamic visual-motor coordination of hands.

Results and Discussion: The devices listed above and their combinations are presently used in research and clinical medical organizations. Collaboration with leading medical organizations of the Russian Academy of Medical Sciences and the Ministry of Health of the Russian Federation is an essential component of successful work. Consider several examples.

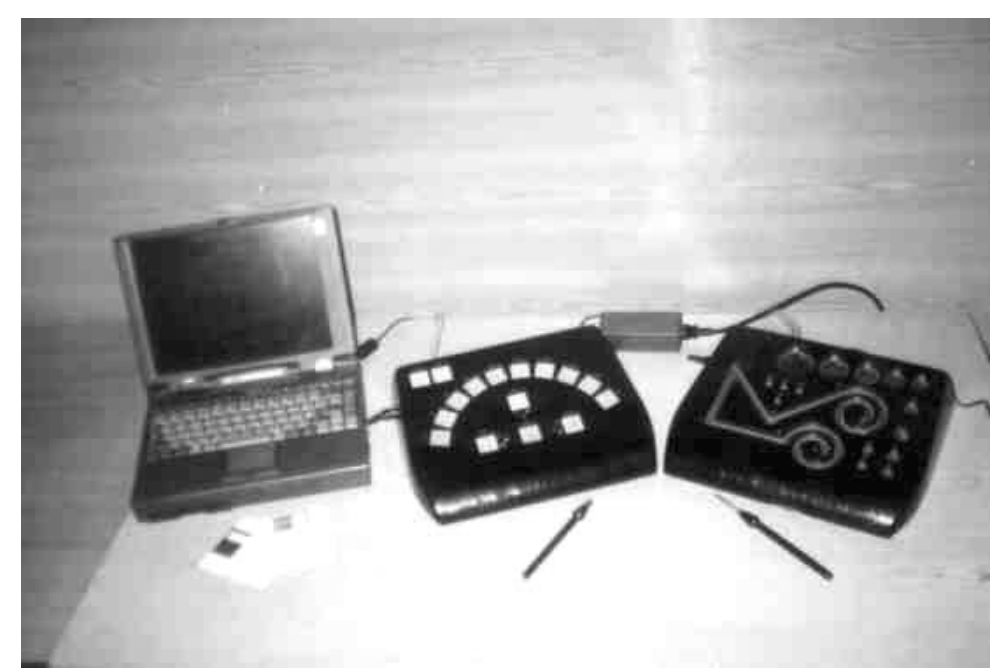

Fig. 1. The PSYCHOMATH psycophisiological research system 
A group of pediatric patients (ages from 3-4 to 14-16 years) with various psychoneurological disorders (epilepsy, mental retardation, persistent headache, infantile cerebral paralysis (ICP), etc.) as well as a group of apparently healthy children of the same age (Scientific-Research Institute for Pediatrics, Russian Academy of Medical Sciences) were tested using these methods and devices. The results of these studies supported the conclusion of high efficiency of computer-assisted instrumental psychophysiological methods of qualitative and quantitative assessment of age-related changes in the higher cortical functions in children and adolescents and disorders of these functions in patients with different diseases. Pediatric patients with ICP were tested with these devices. The efficacy of treatment was monitored by the parameters of dynamic visual-motor coordination and postural stability. Methodological recommendations for the use of these devices in medical practice were given.

The recommendations for the Ritmotest device were tested at the Municipal Children's Psychiatric Hospital No. 18.

The Ritmotest device was tested at the Burdenko Scientific-Research Institute for Neurosurgery, Russian Academy of Medical Sciences in patients with cerebro-cranial injuries.

Pediatric patients with ICP were tested with the Ataksitest devices at the Research Center for Psychoneurological Disability of Children. The efficacy of treatment was monitored by the parameters of dynamic visual-motor coordination and postural stability.

Conclusion: There is now rapid progress in these technologies. Virtually all branches of medicine use modern computer systems. However, psychoneurology (child psycho-neurology, in particular) is not sufficiently advanced in the context of engineering support. Thus, development of computer technologies for objective assessment of the mental health state of children is considered at the Medical Engineering Research Institute, as a top priority problem.

\section{DSC(Digital Scan Converter)-error-free beamforming method for ultrasound imaging}

Jae-sub Hwang, Tai-kyong Song

Department of Electronic Engineering, Sogang University, C.P.O. Box 1142, Seoul 100-611, Korea

Introduction: Nowadays, the digital scan converter (DSC) has become an indispensable component in real-time ultrasound sector scanners. Hence the mapping process between the polar sampling and the Cartesian display with DSC cannot be a one-to-one function, and the scan conversion process with a finite number of beam lines inevitably results in some unavoidable artifacts in the ultrasound image [1]. So far all the approaches for receive beamforming have been optimized to image the actual and/or virtual beam lines located on polar coordinates [2]. This means that the artifacts associated with existing beamforming techniques are unavoidable unless we sacrifice the frame rates. In this letter, a novel DSC-error-free beamforming technique, named display-pixel-based-focusing (DPBF) by the authors, is proposed which can completely eliminate all the artifacts.

Methods: Fig. 1 describes how the ultrasound waves are focused at the display pixels in the receive focusing stage. Unless otherwise noted, all studies are conducted with a convex array with radius R, maximum view angle $\theta_{\max }$, scanlines N, and view depth D. The box in Fig. 1 indicates the actual size of ultrasound image in the monitor, and consists of $N_{x} \times N_{y}$ display pixels. Consider the case that the receive focusing is directly performed at a pixel $\left(x_{i}, y_{j}\right)$ on the Cartesian coordinates of the display monitor. Here the indices must satisfy both $1 \leq i \leq N_{x}$ and $1 \leq j \leq N_{y}$. First, we obtain a virtual/synthetic 
scanline linking a pixel $\left(x_{i}, y_{j}\right)$ to the origin $\left(x_{A}, y_{A}\right)$. Using this scanline, we can get the nearest neighbor actual/physical scanline on the polar coordinates which satisfies the following condition.

$$
\operatorname{Min}\left|\theta_{x_{i} y_{j}}-\theta_{k}\right|, 1 \leq k \leq N
$$

Fig. 1 shows that the $n$-th actual scanline satisfies the condition in Eq. (1) for the pixel $\left(x_{i}, y_{j}\right)$.

In the DPBF system, the dynamic frequency tracking feature [3], envelope detection, and logarithmic compression as well as dynamic receive focusing are performed at an arbitrary pixel $\left(x_{i}, y_{j}\right)$ on the monitor directly.

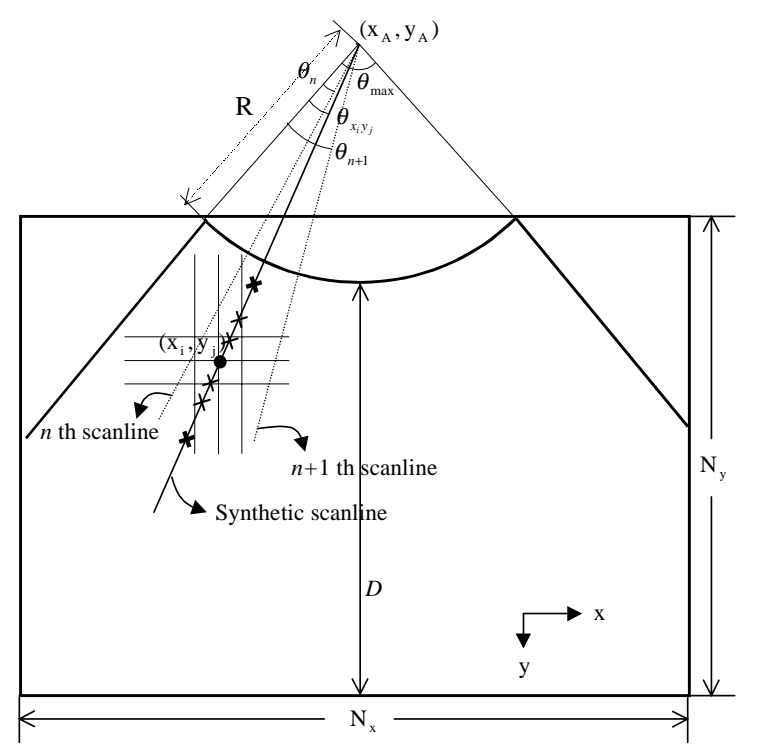

Fig. 1: Illustration of the DPBF method.

To implement the dynamic frequency tracking feature and envelope detection, a few additionally focused radio-frequency (RF) data, which are located around the pixel $\left(x_{i}, y_{j}\right)$ back and forth on the same synthetic scanline, are necessary [3], as depicted in Fig. 1.

Experiments: Experiments are performed with a commercial ultrasound scanner (model SA-8800, Medison Co. Ltd., Korea) modified to allow digitization of the RF signal. Fig. 2 shows magnified ultrasound B-scan images by the read zoomed conventional focusing method (Fig. 2(a)) and proposed DPBF method (Fig. 2(b)) using the $7.5 \mathrm{MHz}$ linear array with $38.4 \mathrm{~mm}$ array length and 128 elements. In Fig. 2, the depth of view is $10 \mathrm{~mm}$ at a $10 \mathrm{~mm}$ distance from the surface of the transducer. Consider $N_{x}=$ $800, N_{y}=688$, and 256 scanlines. In-vivo data used were acquired from a 34-year male neck, and a section of the right thyroid is clearly visible in Fig. 2. 


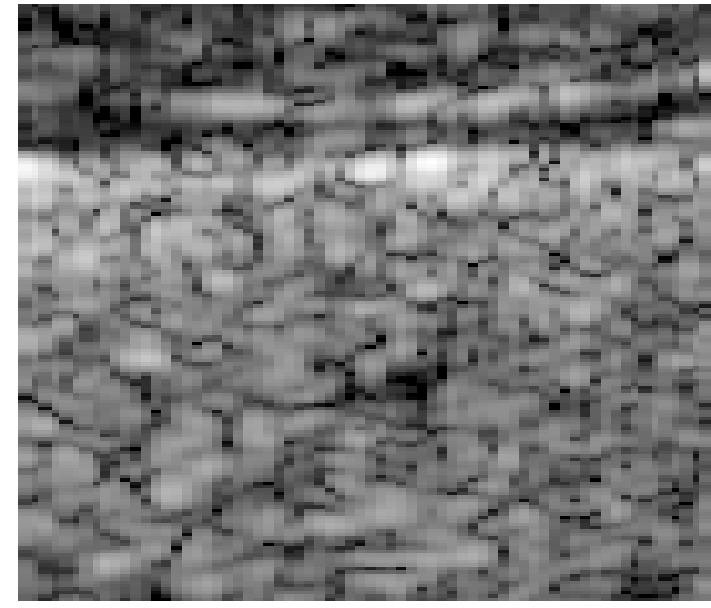

(a)

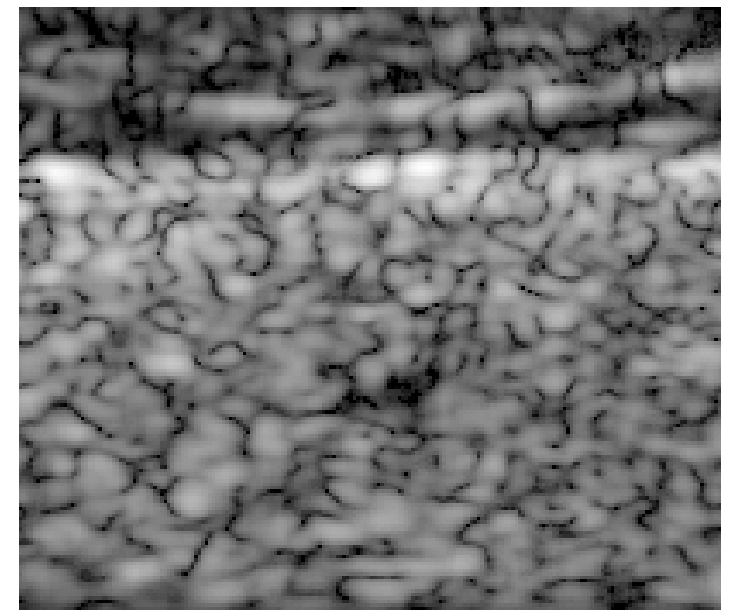

(b)

Fig. 2: Magnified ultrasound B-scan images of the human thyroid using linear array transducer: (a) Conventional read zoom method and (b) DPBF technique.

Results and discussion: Fig. 2(a) closely shows the degradation effect due to the DSC interpolation. In this case, the physical inter-scanline interval is $0.15 \mathrm{~mm}$, and the inter-pixel distance becomes $0.015 \mathrm{~mm}$. The read zoomed image of conventional focusing method, Fig. 2(a), looks quite blocky with DSC. It also is a coarse and rough ultrasound image of the soft tissue in the thyroid. However, Fig. 2(b) shows a quality ultrasound image of high resolution because the ultrasound waves are directly focused at the display pixels on the monitor, rather than sampling points on the polar coordinates. The soft tissues in thyroid are vividly represented with smooth outlines and fine structures. The primary potential clinical benefits of the processing schemes proposed here may include increasing their detectability even with the zoom operation and for the wide view angle especially in OB/GYN applications.

\title{
References
}

[1] M.H. Lee, J.H. Kim, S.B. Park. IEEE Trans., MI-5: 96-105 (1986).

[2] R.A. Mucci. IEEE Trans., ASSP-32: 548-558 (1984).

[3] J.A. Zagzebski. Essentials of ultrasound physics, Mosby-Year Book, Inc. (1996)

\section{Application of indicator dilution method for noninvasive cardiac output measurement}

\author{
Artur Szczepanski $^{\mathrm{a}}$, Jacek Jastrzebski ${ }^{\mathrm{b}}$, Maciej Guc ${ }^{\mathrm{b}, \mathrm{c}}$, Tadeusz Palko ${ }^{\mathrm{d}}$, Andrzej Ksiazkiewicz ${ }^{\mathrm{d}}$ \\ ${ }^{a}$ Institute of Fundamental Technological Research, Polish Academy of Sciences, Poland \\ ${ }^{b}$ Department of Anaesthesia and Intensive Therapy, Medical Center of Postgraduate Education, Warsaw, \\ Poland \\ ${ }^{c}$ Institute of Biocybernetics And Biomedical Engineering, Polish Academy of Sciences, Poland \\ ${ }^{d}$ Institute of Precision and Biomedical Engineering, Faculty of Mechatronics, Warsaw University of \\ Technology, Poland
}

Introduction: The thermodilution method is a standard diagnostic tool for cardiac output determination; however, the measurement is both expensive and invasive [1]. The need for cardiac output determination arises usually when heart and circulation system are in bad condition. The introduction of a Swan-Ganz catheter could be dangerous for patient in ICU, and its use is time limited. This is avoided in our method, 
in which the indicator, hypertonic saline, is injected in a vein, and the dilution curve is determined by means of an external rheometric measurement.

Method: The measurement of the dilution curve is done by means of standard external rheometry with tetrapolar electrodes applied over the carotid artery. 3-10 $\mathrm{ml}$ of $10 \% \mathrm{NaCl}$ solution, or the same amount of distilled water has been used as indicator. The central vein canula is the best injection site, however, if this is not available, the indicator can injected into a peripheral vein as well. The indicator induced impedance changes are small relative to the total measured impedance, so within an expected $1 \%$ accuracy, the cardiac output is:

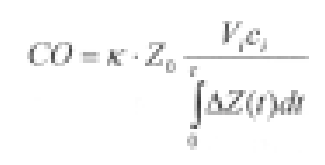

where:

$\mathrm{CO}$ - cardiac output,

$\mathrm{Z}_{0}$ - impedance measured before injection,

$\kappa$ - scaling factor, $\mathrm{V}_{\mathrm{i}}$ - volume injected,

$\mathrm{c}_{\mathrm{i}}$ - concentration of saline relative to blood of bolus injected,

$\Delta \mathrm{Z}(\mathrm{t})$ - recorded dilution curve,

$\tau-$ is corresponding to the peak value of the dilution curve.

The integration is cut at $\tau$ to avoid recirculation effects.

Results and Discussion: We have found that most promising is positioning of electrodes on the carotid artery. It is easy to find its position and even in worst condition of patient substantial part of blood is circulating this way.

The saline indicator losses in lungs [2] may be compared with those of water, which could be used as a tool for evaluation of lung vessels permeability.

\section{Acknowledgements}

This work was supported by State Committee for Scientific Research grant No. 8T11E03515.

\section{References}

[1] T. Pa ko, Cardiac output computer based on the thermodilution method, Post. Fiz. Med. 1983, 18, 4.

[2] W.D. Voorhees III, L.A. Geddes; Right heart output using sodium chloride indicator. IEEE/NSF Symposium on biosensors $-1984,20-23$

[3] A. Szczepa ski, J. Jastrz bski, T. Pa ko, A. Ksi kiewicz, M. Gu ; A new noninvasive method of cardiac output determination. Principles of measurement. Biocybernetics and Biomedical Engineering, 2000, 2, 103-110

\section{Limits of spline interpolation in event related potential mapping}

N. Chauveau, X. Franceries, B. Doyon, B. Rigaud, J.P. Morucci and P. Celsis INSERM Unit 455, Neurology Department, Purpan Hospital Toulouse, France 
Introduction: Event related potentials (ERP) are used as investigation tools in cerebral activation elicited by stimuli presented under different sensory modalities (visual, auditory, etc). ERP mapping is a technique widely used in cognitive processes. A limited number of electrodes reduces the quality of the image. Spherical spline interpolation has been reported as a possible solution to improve the spatial resolution of the averaged maps by correct estimation of the potential values between the electrodes. We present here the limits of this type of interpolation as a function of the electrode number and noise level in the signal.

Method: The three shell sphere head model, as proposed by Perrin et al [1], is implemented with Matlab, using a current dipole with the following characteristics:

Origin and orientation: $\theta=45^{\circ}$ and $\varphi=45^{\circ}$. Eccentricity: 0.8, module: 1 E-8 A.m.
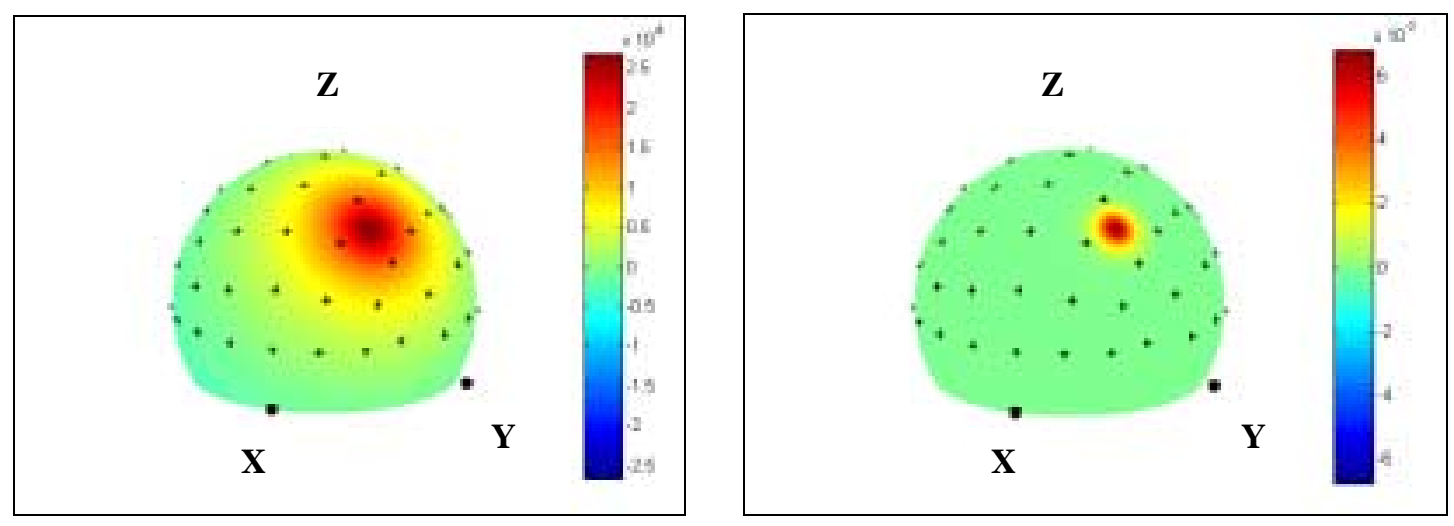

Fig 1: Simulations of voltage and scalp current density maps. The chosen dipole position corresponds to one of the most difficult cases and is not pointing at any electrode.

Equations 1 and 2 give the analytic potential (V) and scalp current density (SCD) and figure 1 shows the corresponding simulated maps with $120 \times 360$ points (1 point per degree).

$$
\begin{aligned}
& \mathrm{V}\left(\mathrm{R}_{\text {scalp }}, \theta, \phi\right)=\frac{1}{4 \pi \sigma \mathrm{R}_{\text {scalp }}^{2}} \sum_{\mathrm{n}=1}^{\infty} \xi \mathrm{f}^{\mathrm{n}-1}\left[\frac{(2 \mathrm{n}+1)^{3}}{\mathrm{n}(\mathrm{n}+1) \mathrm{d}_{\mathrm{n}}}\right]\left[\mathrm{nM}_{\mathrm{R}} \mathrm{P}_{\mathrm{n}}(\cos \theta)+\mathrm{M}_{\mathrm{T}} \mathrm{P}_{\mathrm{n}}^{1}(\cos \theta) \cos \phi\right] \\
& \operatorname{SCD}\left(\mathrm{R}_{\text {scalp }}, \theta, \phi\right)=\frac{1}{4 \pi \mathrm{R}_{\text {scalp }}^{4}} \sum_{n=1}^{\infty} \xi \mathrm{f}^{\mathrm{n}-1}\left[\frac{(2 \mathrm{n}+1)^{3}}{\mathrm{~d}_{\mathrm{n}}}\right]\left[\mathrm{nM}_{\mathrm{r}} \mathrm{P}_{\mathrm{n}}(\cos \theta)+\mathrm{M}_{\mathrm{t}} \mathrm{P}_{\mathrm{n}}^{1}(\cos \theta) \cos \phi\right] \\
& \text { where } \mathrm{d}_{\mathrm{n}}=[(\mathrm{n}+1) \xi+\mathrm{n}]\left[\frac{\mathrm{n}}{\mathrm{n}+1} \xi+1+(1-\xi)\left(\mathrm{f}_{1}^{2 \mathrm{n}+1}-\mathrm{f}_{2}^{2 \mathrm{n}+1}\right)\right]-\mathrm{n}(1-\xi)^{2}\left(\frac{\mathrm{f}_{1}}{\mathrm{f}_{2}}\right)^{2 \mathrm{n}+1}
\end{aligned}
$$

where $\xi, \mathrm{f} 1 \mathrm{f} 2$ and $\mathrm{f}$, the eccentricity, are defined by:

$$
\xi=\frac{\sigma_{2}}{\sigma}=\frac{0.0042}{0.33} \text { and } \mathrm{f}_{1}=\frac{\mathrm{R}_{\text {brain }}}{\mathrm{R}_{\text {scalp }}} \quad \mathrm{f}_{2}=\frac{\mathrm{R}_{\text {bone }}}{\mathrm{R}_{\text {scalp }}} \quad \mathrm{f}=\frac{\mathrm{z}}{\mathrm{R}_{\text {scalp }}}
$$

$\sigma$ and $\sigma_{2}$ are respectively the brain and bone conductivity, $P_{n}$ and $P_{n}^{1}$ are the Legendre and associated Legendre polynomials of order 1.

A small subset corresponding to EEG caps $(29,61,120$ and 397 electrodes) is extracted from the 43200 simulated values. Noise is added to the electrode potential values before interpolation. The level of 
noise is defined as a percentage of the maximum voltage map value and is randomly uniform applied to the electrodes. Different numbers of electrodes and different levels of noise were simulated.

Two indices $\mathrm{MAG}$ and $\mathrm{RDM}$ are used to qualify the fitting quality. $\mathrm{MAG}=\sqrt{\frac{\sum_{\mathrm{i}=1}^{\mathrm{N}} \mathrm{V}_{\mathrm{Pi}}^{2}}{\sum_{\mathrm{i}=1}^{\mathrm{N}} \mathrm{V}_{\mathrm{Si}}^{2}}}$ $\mathrm{RDM}=\sqrt{\sum_{\mathrm{i}=1}^{\mathrm{N}}\left[\frac{\mathrm{V}_{\mathrm{Si}}}{\sqrt{\sum_{\mathrm{i}=1}^{\mathrm{N}} \mathrm{V}_{\mathrm{Si}}^{2}}}-\frac{\mathrm{V}_{\mathrm{Pi}}}{\sqrt{\sum_{\mathrm{i}=1}^{\mathrm{N}} \mathrm{V}_{\mathrm{Pi}}^{2}}}\right]^{2}}$

$\mathrm{S}$ for simulated

P for interpolated

$\mathrm{N}$ : point number (43200)

MAG is a magnification factor (optimal value 1) between simulated and interpolated values. RDM is an index (optimal value 0) which compares the distribution of the data independently of any MAG effect:

Results and discussion: Results in figure 2 show that MAG and RDM are good up to $10 \%$ noise with all caps. In this case the lowest RDM is obtained with a 120 electrode cap. An important increase both for MAG and RDM can be observed for a 397 electrode cap.
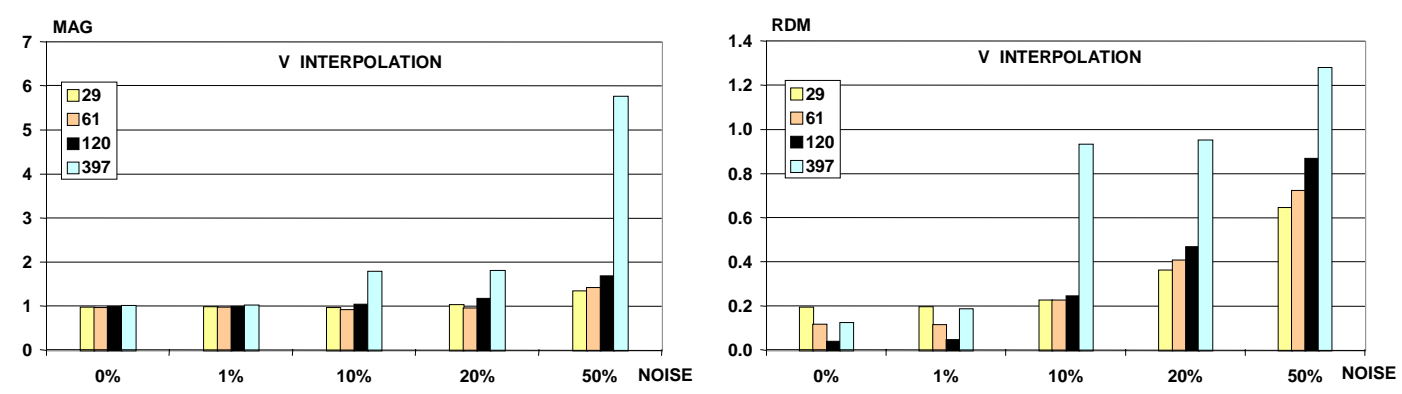

Figure 2: MAG and RMS indices for different head caps and different noise levels obtained with the chosen current dipole.

In conclusion, the best compromise seems to be the use of around 120 electrodes. A higher electrode number increases both MAG and RDM values.

\section{References}

[1] F. Perrin, J. Pernier, O. Bertrand, J.P. Echallier, "Spherical splines for scalp potential and current density mapping, Electroencephalogr. Clin. Neurophysiol., 1989, 72, pp 184-187.

[2] G. Wahba, "Spline interpolation and smoothing on the sphere, "Siam J. Sci. Stat. Comp., 1981, 2(1), pp 5-16.

\section{Selected algorithms for the computer analysis of microscope scans in the system for early detection} of the urinary bladder cancer

P. Jaszczak, A. Dulewicz, A.Nechaj, D. Pietka

Institute of Biocibernetics and Biomedical Engineering PAS, Warsaw, Poland 
Introduction: The concept of the system for the support of the early diagnosis of the urinary track tumors is as follows: The data for the analysis is acquired in a non invasive manner from the patient's urine. Microscope specimen containing appropriately colorized nuclei of the cells of the urinary track epithelium is scanned under the microscope with the help of the computer controlled motorized scanning table. The image is then registered by a microscope coupled TV camera and transmitted to the computer through the image acquisition adapter. The result of the image processing and computer analysis of the acquired image is a preliminary classification on the basis of the extent of the cancer changes detected in the specimen.

On the basis of the concept presented a computer system named DIPS has been created. In the following section the structure and selected algorithms of the system are presented.

Image acquisition: Dedicated software controls the motorized scanning table mounted under the lens of the optical microscope. A set of algorithms for precise scanning of the whole or selected parts of the specimen has been worked out. Also a pre-scanning routine, which builds a map of the specimen for the following processing stages has been worked out and implemented.

Initial processing: A set of algorithms for the improvement of both subjective and objective image quality has been implemented. The most crucial methods of objective image quality improvement are: specimen lighting equalization, brightness normalization and image noise removal.

Objects extraction: In the DIPS system a set of standard methods for objects extraction have been implemented - like Sobel's filters for instance. A specialized algorithm, which is multiple times faster than the filtration methods and gives very good results in the class of images that are in the scope of the system has been worked out. The algorithm relies on image thresholding with the threshold level selected automatically on the basis of image histogram analysis. Presented below is a characteristic shape of the histogram and the result of the algorithm application:

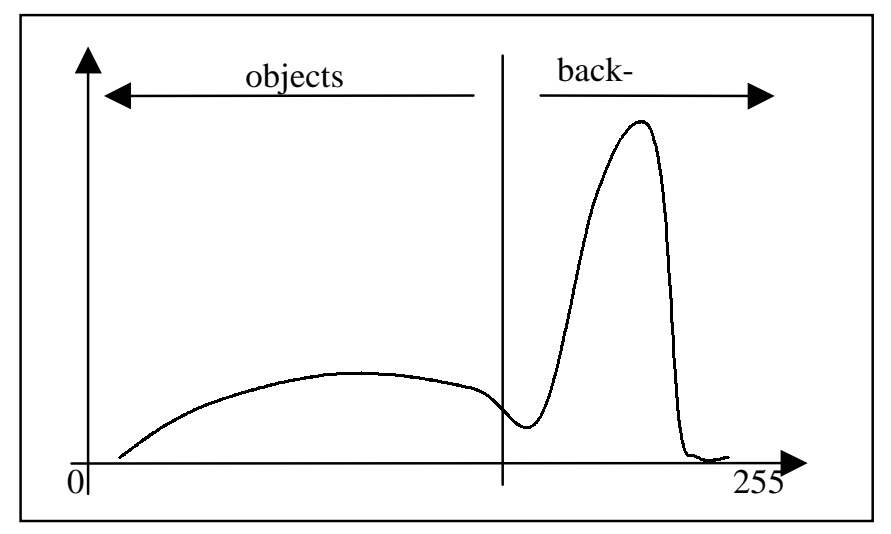

Fig 1. A typical histogram 
(a)

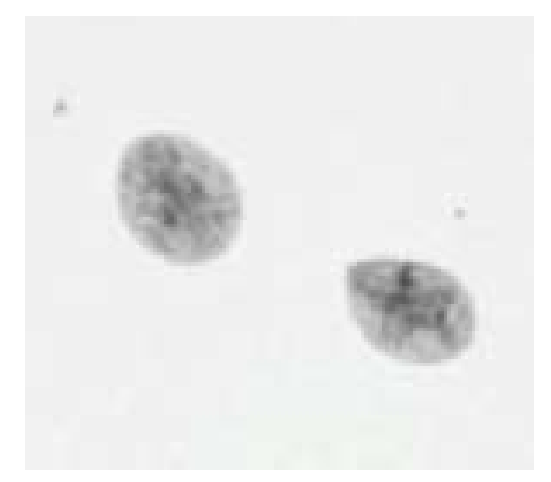

(b)

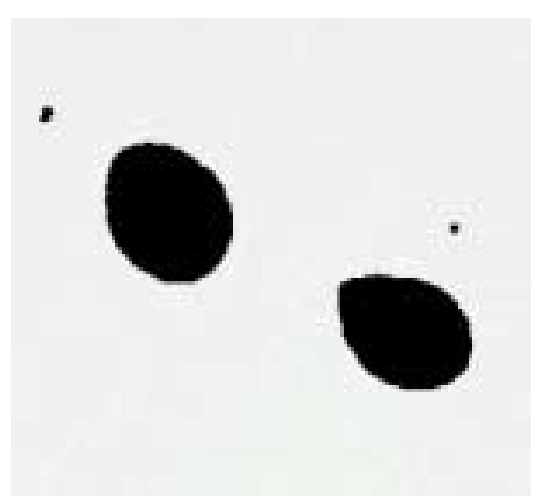

Fig 2. Objects extraction: a) original image, b) image after thresholding process

Completion of the extraction algorithms are the undesirable objects (artifacts) removal algorithms. For this purpose a set of algorithms has been worked out. Worth of mentioning here are the algorithms, which remove artifacts basing on: size, overlapping with another objects and contact with the borders of the observation field.

Object's parameters measurement: For each of the objects selected a set of parameters describing it's features is calculated. An algorithm has been worked out, which calculates a set of parameters in the following categories:

- geometric parameters - describe geometric characteristic of the objects such as: area, perimeter, shape, location and orientation.

- statistical textural parameters - describe the characteristic of the object in the quantitative manner - maximum luminosity, average luminosity, integrated optical density.

- topological textural parameters - describe location of the regions of different brightness inside the object - moment of inertia.

Classification: On the basis of the parameters measurement nuclei are classified to an appropriate group: "control", G1, G2, G3. The group "control" represents images characteristic to a healthy patient, whereas the groups G1, G2 and G3 contain images characteristic to a different stages of the pathological changes.

Conclusion: All of the mentioned techniques and algorithms have been implemented to meet the requirements of the DIPS system. An experiment has been carried out with the system where a set of specimen taken both from healthy people and with different stages of the pathological changes has been presented for measuring and classification. The total of 23 healthy and 35 ill patients have been investigated. A result of 95.65 percents classified correctly to the "control" group and 94.28 percents classified correctly to the pathological groups has been achieved. Such a good result has been achieved mainly thanks to precise choice of the nucleus images taken for the experiment. It should be taken into account that in the routine investigations will not probably be this good. The results achieved encourage to the continuation of the development of the system.

The project has been carried out in the Department of Biomedical Information Processing Methods in the Institute of Biocibernetics and Biomedical Engineering PAS in the collaboration with the Department of Histology and Embryology, Medical Academy of Warsaw. 
References

[1] Dulewicz A.: „Sprawozdanie merytoryczne z projektu badawczego Nr 8 T11E 019 08”, IBIB PAN, 1997

[2] Dulewicz A., Pi tka D.: „Komputerowa analiza obrazów cytologicznych w diagnostyce nowotworów dróg moczowych”, IBIB PAN, 1994

[3] Watkins D.: „Nowoczesne metody przetwarzania obrazu”, WNT, 1995

\title{
Digital HDTV for Improved Image Quality of Endoscopes
}

\author{
P. Niederer ${ }^{\mathrm{a}}$, J. Häfliger ${ }^{\mathrm{a}}$, P. Blessing ${ }^{\mathrm{a}}$, Y. Lehareinger ${ }^{\mathrm{a}}$, D. Doswald ${ }^{\mathrm{b}}$, N. Felber ${ }^{\mathrm{b}}$ \\ anstitute of Biomedical Engineering, University of Zurich and Swiss Federal Institute of Technology, \\ Zurich, Switzerland \\ ${ }^{\mathrm{b}}$ Integrated Systems Laboratory, Swiss Federal Institute of Technology, Zurich, Switzerland
}

Abstract: Medical endoscopy has become an indispensable tool for the purpose of minimally invasive diagnostic and therapeutic procedures. An endoscope is intended to facilitate an external observation of a location inside the human body and thereby cause minimal traumatization. For this purpose, a natural or a surgically prepared small opening of the human body is utilized to insert the tip of the endoscope and to advance it to the location of interest.

A standard television system is usually attached to the distal end of an endoscope for image acquisition and presentation. Of primary importance for the user are image quality, ease of application and safety. This communication is primarily concerned with aspects relating to image quality. The optical properties of various types of endoscopes are analysed on the basis of measurements and simplified theoretical considerations. The relevant optical characteristics thereby include the spatial, dynamic and temporal resolution as well as image distortion and homogeneity of brightness. It is found that the inherent optical quality, in particular with respect to spatial resolution of most endoscopes exceeds the performance of standard TV in part substantially. Accordingly, a special purpose advanced digital television system exhibiting high spatial resolution, furthermore true color and high contrast performance has been developed. Since endoscopic images usually are circular, its aspect ratio is quadratic with a spatial resolution of $1000 \times 1000$ pixels. The temporal resolution is variable from $1 \mathrm{~Hz}$ to $30 \mathrm{~Hz}$ noninterlaced. Some design aspects and performance of the system are shown.

An autofocus as well as an automatic illumination control are furthermore desirable features of an endoscope, because they relieve the user from performing a task which can be automated and thereby prevents unnecessary interruptions in the work to be performed. Thanks to the fact that our new HDTV system operates on a fully digital basis, a useful and stable solution for both tasks could be found. A new passive autofocus procedure has been developed which exhibits a stable operation in real time under all circumstances of interest. It is in particular more sensitive, has a larger SNR than other focus criteria known to the authors, exhibits no secondary extrema which could adversely affect proper focusing and is independent of the image content. Automatic illumination control, in turn, should ascertain an adequate illumination of the area of interest and be insensitive to reflections of surgical instruments. An empirical procedure has been found to solve this problem.

\section{The new approach to evaluation of vasovagal syncope patients treated by pacing of the heart}

Kazimierz Peczalski, Dariusz Wojciechowski, Piotr Sionek, Marek Kowalewski Laboratory of Clinical Bioengineering IBIB PAS \& Wolski Hospital Warsaw, Poland 
Introduction: The treatment of recurrent syncope of unknown aetiology becomes a severe problem in today medicine. The standard classification of vasovagal syncope proposed by Sutton [1] consists of four types of vasovagal reaction (including cardiodepressive reaction i.e heart rate decrease, and vasodepressive reaction i.e. arterial pressure decrease): 1.mixed, 2a.cardiodepressive, $2 \mathrm{~b}$..cardiodepressive, 3. vasodepressive. The occurrence timing of changes of measured parameters during syncope is crucial for diagnosis and proper treatment evaluation. For some vasovagal syncope patients the cardiac pacing with a special algorithm for rate drop response is applied.

Method: The head-up tilt test (HUT) is very often used for the recurrent syncope diagnosis.

HUT usually is performed in the morning hours on a tilt table with a patient foot plate support. Patients should be in fasting state and the concomitant medication cancelled at least 5 half-lives prior to study. Informed consent is obtained from each patient. The travel time between 0 and 75 degrees is approximately 10 seconds. The study room should be quiet and isolated.

In this test the electric and hemodynamic activity of the heart and the electric brain activity are measured simultaneously, beat-to-beat (real time) recorded. ECG and EEG are continuously monitored and blood pressure is taken automatically for every beat of the heart by applying the Penaz plethyzmographic method.

After approximately 30 minutes of observation in horizontal position patient is tilted to a 75 degree upright position for 45 minutes. The positive HUT is defined as the occurrence of syncope or pre-syncope in the upright position with hypotension or bradycardia, or combined form of both. If HUT test is positive, patient is returned to supine position When syncope occurs in horizontal position or syncope occurs without hypotension and bradycardia a non-diagnostic result is defined.

System Description: The system we introduced consists of two modules: electrophysiological and hemodynamic: i.e. ECG (analysis of dysfunctions of sinoatrial and atrioventricular nodes and heart rate variability), and EEG (disturbances of brain electrical activity) done together with Portapress [2] (continuous measurements of arterial pressure, stroke volume and cardiac output). The crucial point is a non-invasive continuous measurement of arterial blood pressure by applying the Penaz plethyzmographic method [3].

Discussion: The measurement system for HUT examination constructed in our laboratory consists of two modules: electrophysiologic and hemodynamic. Recorded data are used for evaluation of patients according to criteria given by Sutton et al.. Commonly used methods of recording and analysis are applied for the electrical activity of brain and heart examination. However, new algorithms of heart rate variability evaluation would be helpful in the more precise diagnosis of vasovagal system disturbances. The method of arterial pressure measurements, commonly used in medicine, is insufficient in the vasovagal syncope examination. The invasive monitoring of arterial blood pressure provided by caniulas inserted into patient's arteries can influence his mental/emotional state of the patient and change test results. The non-invasive cuff fixed on the patient arm used by other non-invasive methods does not provide beat-to-beat recording of arterial pressure. During the measurement process the cuff must be inflated up to the level of the arterial pressure. When applied, it often can cause the ischemic problems of the arm. Therefore, in our system, the Portapress unit using the arterial volume clamp method of J. Penaz and the physiological criteria of $\mathrm{K}$. Wesseling is chosen. The preliminary study done in 20 patients has shown that the timing between heart rate fall and arterial pressure fall prior to syncope and during syncope onset as determined of pacing treatment effectiveness. For evaluation of syncope patients for proper pacing treatment the new timing criteria have to be worked out in a bigger group of patients. The EEG module introduced by our team to the system provides the timing of onset of the brain electrical activity disturbances prior to syncope. The brain disturbance is detected by brain ischemic incidence 
during HUT - slow waves propagation. Based on the preliminary results of pacing the effectiveness the EEG parameter should also be recognised in new evaluation criteria.

\title{
References
}

[1] R.Sutton, M.Petersen, M.Bringole, A.Raviele, C.Menozzi, P.Giani. Proposed classification for tilt induced vasovagal syncope. Eur.J.C.P.E. 1992; 3:180-183.

[2] Portapress User's Guide. TNO, Amsterdam, Netherlands, 1999,

[3] Kazimierz P czalski Dariusz Wojciechowski, , Piotr Sionek, Marek Kowalewski. Diagnostic of recurrent syncope system for electrophysiological parameters measurement. Wave methods \& Mechanics in Biomedical Engineering. Cracow, Poland. 2000: 147-150..

\section{Localization of component parts of retinal images}

\author{
Mohsen Farid ${ }^{\mathrm{a}, \mathrm{b}}$, Fionn Murtagh ${ }^{\mathrm{b}}$ \\ ${ }^{a}$ Dundalk Institute of Technology, Dundalk, Ireland \\ ${ }^{b}$ Queen's University of Belfast, Northern Ireland
}

We explore a range of methods for localizing optic disk, fovea and retinal blood vessels from digital fundus images. In particular we investigate multiscale transforms for edge detection, and for feature finding on appropriate resolution scales. Shown below are (i) one grayscale retinal image, (ii) a weighted sum of second derivative edge map using resolution level 3 of a wavelet transform (B3 spline à trous) and the original data, providing a visualization of the blood vessels in particular, and (iii) scale 2 from a multiresolution median transform (Starck et al., 1998), again aiding the tracing of blood vessels. Further aspects of the system proposed in Sinthanayothin et al. (1999) will be assessed in terms of providing a robust and stable processing pipeline.

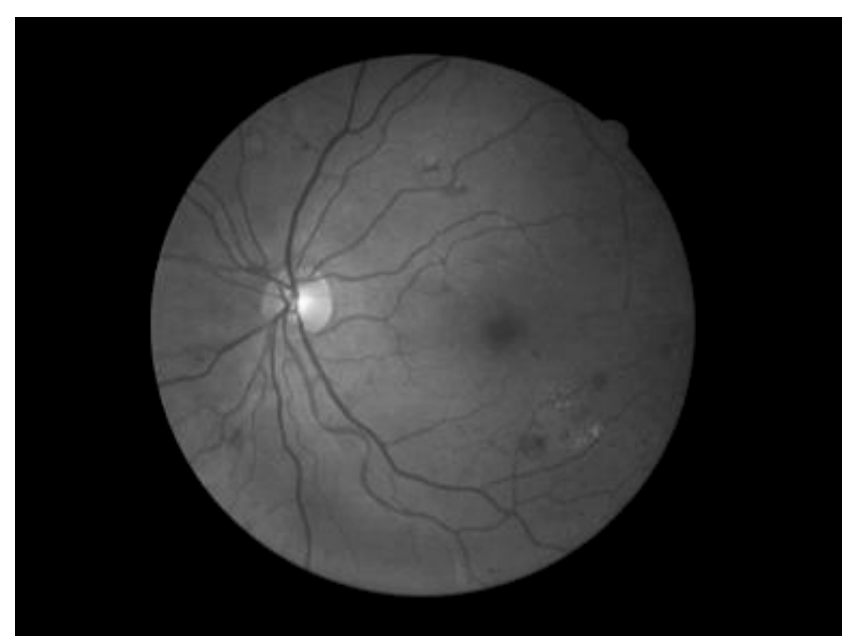

Original image, one colour. 


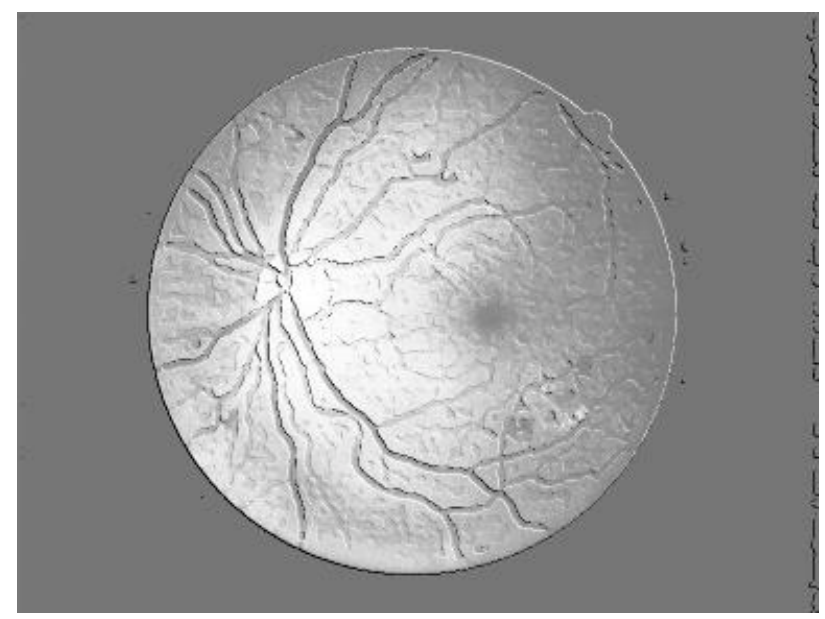

Second derivative map of scale 3 of a wavelet transform (B3 spline à trous transform).

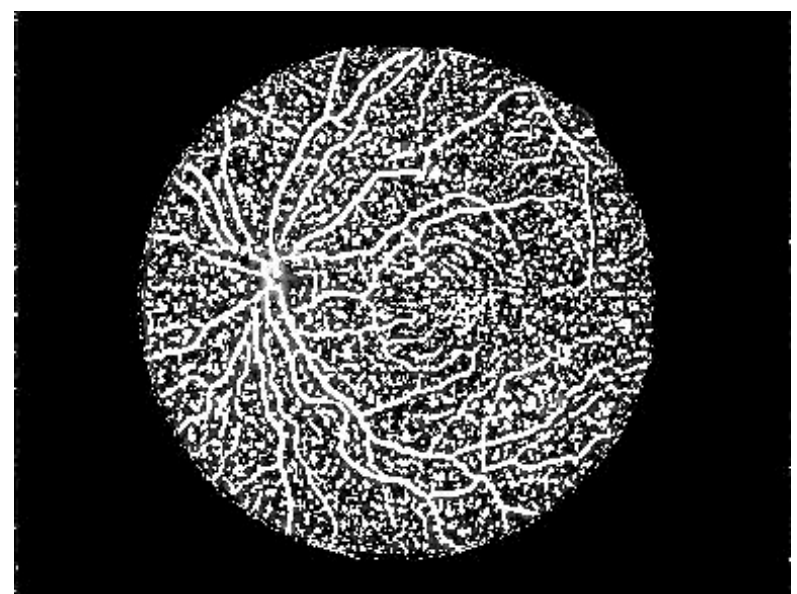

Scale 2 from a multiresolution median transform.

\section{References}

[1] Chanjira Sinthanayothin, JF Boyce, HL Cook and TH Williamson, Automated localisation of the optic disc, fovea, and retinal blood vessels from digital colour fundus images, Br J Ophthalmol 1999, 83: 902-910

[2] JL Starck, F Murtagh and A Bijaoui, Image and Data Analysis: The Multiscale Approach, Cambridge University Press, 1998 